\title{
On the three-dimensional configuration of coronal mass ejections
}

\author{
H. Cremades and V. Bothmer
}

\author{
Max-Planck-Institut für Sonnensystemforschung ${ }^{\star}$, Max-Planck-Str. 2, 37191 Katlenburg-Lindau, Germany \\ Received 1 December 2003 / Accepted 20 February 2004
}

\begin{abstract}
Coronal mass ejections (CMEs) are a direct consequence of the dynamic nature of the solar atmosphere. They represent fundamental processes in which energy is transferred from the Sun into interplanetary space, including geospace. Their origin, 3D structure and internal magnetic field configuration are to date not well understood. The SOHO spacecraft, launched by the end of 1995, has provided unprecedented data on CMEs since instruments switched on in 1996. From a detailed investigation of the full set of LASCO (Large Angle Spectroscopic Coronagraph) observations from 1996 to the end of 2002, a set of structured CME events has been identified, which exhibits white-light fine structures likely indicative of their internal magnetic field configuration and possible 3D structure. Their source regions in the low corona and photosphere have been inferred by means of complementary analyses of data from the Extreme-Ultraviolet Imaging Telescope (EIT) and Michelson Doppler Imager (MDI) on board SOHO, and ground-based $\mathrm{H} \alpha$ measurements.

According to the results of this study, structured CMEs arise in a self-similar manner from pre-existing small scale loop systems, overlying regions of opposite magnetic polarities. From the characteristic pattern of the CMEs' source regions in both solar hemispheres, a generic scheme is presented in which the projected white-light topology of a CME depends primarily on the orientation and position of the source region's neutral line on the solar disk. The paper also provides information about the white-light characteristics of the analysed CMEs, such as angular width and position angle, with respect to their source region properties, such as heliographic location, inclination and length, including the frequency and variation of these parameters over the investigated time period.
\end{abstract}

Key words. Sun: corona - Sun: coronal mass ejections (CMEs) - Sun: filaments

\section{Introduction}

Coronal Mass Ejections (CMEs) constitute a fascinating subject of investigation in solar physics, since the first images of these transients were obtained in the early 1970's by the coronagraph onboard the Seventh Orbiting Solar Observatory (OSO 7; Tousey et al. 1974) and onboard Skylab (Gosling et al. 1974). Based on the large set of CMEs detected during the Solar Maximum Mission, Hundhausen et al. (1984) introduced the definition that describes a CME as "....an observable change in coronal structure that occurs on a time scale between a few minutes to hours and involves the appearance of a new, discrete, bright, white-light feature in the coronagraph field of view".

Knowledge concerning CMEs has largely grown in the last three decades. However, their three-dimensional configuration still remains unclear, primarily due to the optical thinness of the coronal medium and the highly dynamic nature of CMEs. Since coronagraphs measure mainly the photospheric light scattered by free electrons in the coronal plasma, yielding the integrated density along the line of sight, they only provide us with a particular view of a CME projected on the plane of the sky. To increase the spatial and time resolution of the observations,

Send offprint requests to: $\mathrm{H}$. Cremades,

e-mail: cremades@linmpi.mpg.de

* Formerly Max-Planck-Institut für Aeronomie. progressively sophisticated instruments have been developed and the first simultaneous observations from two different vantage points in space will be provided in the near future by the NASA STEREO mission (http://stp.gsfc.nasa.gov/ missions/stereo/stereo.htm; e.g. Rust 1998; Bothmer et al. 1998).

The so called three-part structure CMEs constitute the most stunning CME events. They appear as having a bright leading edge, a dark void and a bright core (Illing \& Hundhausen 1985) comprised of prominence material. Based on the close association found between CMEs and eruptive prominences/disappearing filaments (Munro et al. 1979), Trottet \& MacQueen (1980) attempted to correlate the magnetic field structure of looplike CMEs with the orientation of $\mathrm{H} \alpha$ filaments and suggested that CMEs are planar loops. Contrary to this result, Webb (1988) found no such correlation in a separate study of filament orientations and looplike CMEs and concluded that CMEs are indeed three-dimensional entities.

Numerous efforts have been directed towards the attainment of 3D MHD models that describe the initiation, expulsion out of the corona, and evolution of the classic three-part structure CME (e.g. Gibson \& Low 1998; Amari et al. 2000). Other approaches focused directly on the analysis of observational properties of CMEs in order to infer whether CMEs can be best described as lightbulb bubbles, arcades comprised of 
loops, or curved flux tubes (e.g. Crifo et al. 1983; Schwenn 1986; MacQueen 1993; Vourlidas et al. 2000; Plunkett et al. 2000; Zhao et al. 2002).

The LASCO Coronagraph onboard the Solar and Heliospheric Observatory (SOHO; Fleck et al. 1995), launched in December 1995, provides to date unprecedented coronal images, due to its high cadence and spatial resolution of the Sun's corona. The observations of CMEs taken since launch (e.g. Howard et al. 1997; St. Cyr et al. 2000; Gopalswamy et al. 2003) constitute a unique dataset to study whether the white-light observations reveal the magnetic structure of CMEs, and can be used to infer their 3D nature. With this purpose in mind, a subset of CMEs has been selected for analysis, which exhibited clear white-light structures, expected to be suggestive of their internal magnetic field configuration and possible 3D structure. Since the unstructured halo CMEs arise from the central portion of the solar disk, structured CMEs are expected to originate from limb or near limb regions, being therefore better tracers of CME structure and dynamics. The understanding of the three-dimensional configuration of CMEs is an essential issue to explore the physical mechanisms that originate them and to model their evolution into interplanetary space, since it is well known that CMEs are the most important causes of space weather disturbances (e.g. Webb 2000).

\section{Instrumentation and data}

The full dataset compiled by the Large Angle Spectroscopic Coronagraph (LASCO; see Brueckner et al. 1995) onboard SOHO from 1996 to the end of 2002 was the starting point for this investigation. The pixel sizes of the $\mathrm{C} 1, \mathrm{C} 2$ and $\mathrm{C} 3$ coronagraphs of LASCO are 5.6, 11.4 and 56 arcsec respectively, and the fields of view (FOV) range from 1.1 to $3 R_{\odot}$ for $\mathrm{C} 1,1.5$ to $6 R_{\odot}$ for $\mathrm{C} 2$ and 3.7 to $30 R_{\odot}$ for $\mathrm{C} 3$, while the typical time resolution is around $12 \mathrm{~min}$. Various formats of data facilitated this study, from daily quick look mpeg movies (http: //star.mpae.gwdg.de/dailympg/), to full resolution images of $1024 \times 1024$ pixels in FITS format.

Data from the Extreme-Ultraviolet Imaging Telescope (EIT) aboard SOHO, described by Delaboudinière et al. (1995), were used to investigate the CMEs' source regions. EIT has a FOV that covers the solar disk and the corona up to $1.5 R_{\odot}$, with a pixel size corresponding to 2.6 arcsec. Images in the line of $195 \AA$ were essential for this work since they provide high cadence observations of dynamic processes in the low corona, crucial when the onset signatures of eruptions are analysed. Additionally, images in the $304 \AA$ line were consulted, to study the association of CMEs with prominence eruptions.

SOHO's Michelson Doppler Imager (MDI; see Scherrer et al. 1995), provides full-disk magnetograms with a resolution of 4 arcsec. Out of the daily images, synoptic charts are constructed for each solar rotation, which are available at http://soi.stanford.edu/magnetic/ index6.html. These magnetic maps of the Sun were used to locate the photospheric source regions of the CMEs and to investigate their field structure.
Ground-based observations from the Observatory of Meudon (http://bass2000.obspm.fr/home.php) and the Big Bear Solar Observatory (ftp://ftp.bbso.njit.edu) provided information on the structure and location of $\mathrm{H} \alpha$ filaments, and the Soft X-Ray Telescope (SXT) on board the Yohkoh satellite was used to examine plasma processes at higher coronal temperatures such as during flares (movies can be generated at http: //wWw . Imsal . com/YPOP/ sxt_movie.html). For additional comparisons with respect to the spatial distribution and size of coronal holes, Kitt Peak He $10830 \AA$ data were employed, available at ftp: //nsokp.nso.edu.

\section{Selection and characteristics of structured CMEs}

In order to understand better the CME 3D morphology, "structured" CME events were identified from the LASCO dataset, during the period of January 1996 through December 2002. We define a "structured" CME as an outward moving whitelight feature in the field of view of $C 2$, that reveals inherent fine structure discernable from the ambient corona, and which develops in the field of view of $C 2$ as part of the entire whitelight feature during its outward motion. Based on this definition, halo and narrow CMEs (see e.g. Yashiro et al. 2003) as well as faint ones typically represent counterexamples to structured CMEs. The June 1998 CME described in Plunkett et al. (2000) and in Srivastava et al. (2000) can be considered as an archetype event. Figure 1 shows two examples of structured CMEs identified in the LASCO dataset in comparison with a CME that is lacking clearly discernable internal white-light structure, and Fig. 2 provides an example for a structured CME observed at the SW-limb on April 23, 2001 in which helical field lines, indicative of a magnetic flux rope structure, seem to overly the trailing prominence material. Events like this are rare because CME cavities are depleted regions, difficult to image in an optically thin medium like the solar corona. They have previously often been misinterpreted as concave outward moving structures after magnetic disconnections as pointed out by Dere et al. (1999).

Daily C2 half-resolution $(512 \times 512$ pix $)$ mpegs served as a baseline for the search of structured CME events. The 276 selected events were compiled in a spreadsheet table, available upon request to the authors. Table 1 contains the most significant columns of this table for the 124 cases for which relevant information about the source regions could be inferred, and which are of prime importance for this study. Figure 3 shows the time distribution of these events together with that of the whole group of 276 structured CMEs, the total number of observed CMEs as taken from Gopalswamy et al. (2003) and the yearly sunspot number for comparison. Note that the subset of structured CMEs represents $4 \%$ of the total amount of observed CMEs, while those with identified source regions represent approximately $2 \%$.

Columns 1 to 4 of Table 1 provide date and time of observation, angular width $(A W)$ and position angle (PA). The CME time was taken from the SOHO LASCO CME Catalog at http: //cdaw . gsfc . nasa.gov/CME_ist/. 
Table 1. Structured CME events and their identified Source Regions selected during the period Jan. 1996-Dec. 2002.

\begin{tabular}{|c|c|c|c|c|c|c|c|c|c|c|c|c|}
\hline \multirow[b]{2}{*}{$\begin{array}{c}\text { Date } \\
\text { (1) }\end{array}$} & \multicolumn{3}{|l|}{$\mathrm{CME}$} & \multicolumn{7}{|c|}{ SR } & \multirow[b]{2}{*}{$\begin{array}{c}\text { Deviation } \delta \\
\text { from source } \\
\text { (deg) } \\
\text { (12) }\end{array}$} & \multirow[b]{2}{*}{$\begin{array}{c}\text { NL-CME } \\
\text { agreement } \\
\text { (13) }\end{array}$} \\
\hline & $\begin{array}{c}\text { Time } \\
(\mathrm{UT}) \\
(2)\end{array}$ & $\begin{array}{c}A W \\
\text { (deg) } \\
(3)\end{array}$ & $\begin{array}{c}\text { Central } \\
\text { PA } \\
(\mathrm{deg}) \\
(4)\end{array}$ & \multicolumn{3}{|c|}{$\begin{array}{c}\text { Carrington coordinates } \\
\text { (deg) }\end{array}$} & $\begin{array}{l}\text { ates } \\
\phi_{\mathrm{C} 2} \\
(8)\end{array}$ & $\begin{array}{l}\text { Tilt from } \\
\text { vertical } \gamma \\
\text { (deg) } \\
\text { (9) }\end{array}$ & $\begin{array}{c}\text { Length } \\
\text { (deg) } \\
(10)\end{array}$ & $\begin{array}{c}\text { Central } \\
\text { PA } \\
(\operatorname{deg}) \\
(11)\end{array}$ & & \\
\hline 1996 Nov. $05^{a}$ & $02: 35$ & 49 & 271 & -15 & 241 & -13 & 239 & 50 & 2.3 & 249 & 22 & $\mathrm{y}$ \\
\hline 1996 Nov. $28^{a}$ & $16: 50$ & 43 & 267 & 1 & 242 & 6 & 241 & 5 & 4.8 & 274 & 7 & $\mathrm{y}$ \\
\hline 1997 Feb. 07 & $00: 30$ & 121 & 247 & -25 & 335 & -47 & 320 & 34 & 25.5 & 223 & 24 & $\mathrm{y}$ \\
\hline 1997 Feb. $23^{a}$ & $02: 55$ & 75 & 88 & 31 & 349 & 37 & 342 & 47 & 9.0 & 57 & 32 & $\mathrm{y}$ \\
\hline 1997 Mar. 09 & $04: 30$ & 66 & 75 & 4 & 208 & 11 & 206 & 17 & 7.1 & 74 & 1 & $\mathrm{nc}$ \\
\hline 1997 Apr. 30 & $04: 50$ & 64 & 83 & 4 & 215 & 17 & 214 & 3 & 13.2 & 78 & 5 & $\mathrm{y}$ \\
\hline 1997 May 05 & $06: 30$ & 41 & 85 & 23 & 143 & 17 & 141 & 19 & 5.5 & 69 & 16 & $\mathrm{nc}$ \\
\hline 1997 Sep. $09^{a}$ & 20:06 & 88 & 278 & 18 & 116 & 33 & 98 & 49 & 22.5 & 307 & 29 & $\mathrm{y}$ \\
\hline 1997 Sep. $20^{a}$ & $10: 20$ & 76 & 269 & -16 & 302 & -33 & 286 & 44 & 22.0 & 246 & 22 & $\mathrm{y}$ \\
\hline 1997 Oct. $12^{b}$ & $06: 26$ & 54 & 255 & -25 & 349 & -32 & 340 & 52 & 10.6 & 240 & 15 & $\mathrm{y}$ \\
\hline 1997 Oct. $16^{b}$ & $22: 29$ & 81 & 261 & -25 & 287 & -35 & 278 & 43 & 12.8 & 239 & 23 & $\mathrm{y}$ \\
\hline 1997 Nov. 14 & $10: 14$ & 78 & 77 & 21 & 111 & 17 & 107 & 39 & 5.7 & 71 & 5 & $\mathrm{nc}$ \\
\hline 1997 Nov. 18 & $08: 27$ & 49 & 280 & 21 & 240 & 37 & 213 & 59 & 28.4 & 300 & 20 & $\mathrm{y}$ \\
\hline 1997 Dec. 06 & $10: 27$ & 144 & 323 & 39 & 295 & 42 & 276 & 81 & 14.7 & 333 & 10 & $\mathrm{y}$ \\
\hline 1998 Feb. $04^{a}$ & $17: 02$ & 84 & 287 & 43 & 305 & 48 & 261 & 84 & 30.8 & 317 & 31 & $\mathrm{y}$ \\
\hline 1998 Feb. 23 & $02: 55$ & 59 & 254 & -29 & 30 & -22 & 29 & 9 & 6.7 & 245 & 9 & $\mathrm{nc}$ \\
\hline 1998 Feb. 24 & $07: 28$ & 38 & 91 & 29 & 202 & 19 & 200 & 10 & 10.0 & 66 & 25 & $\mathrm{nc}$ \\
\hline 1998 Mar. $23^{a}$ & 09:33 & 68 & 265 & -19 & 34 & -27 & 24 & 50 & 12.6 & 246 & 19 & cs \\
\hline 1998 Apr. 14 & $05: 26$ & 56 & 76 & 16 & 290 & 28 & 283 & 33 & 13.2 & 66 & 10 & $\mathrm{y}$ \\
\hline 1998 Apr. $20^{a, b}$ & $10: 07$ & 125 & 264 & -22 & 31 & -59 & 9 & 31 & 40.7 & 228 & 36 & $\mathrm{y}$ \\
\hline 1998 Apr. $23^{a}$ & $05: 27$ & 113 & 98 & -21 & 140 & -15 & 137 & 24 & 6.8 & 110 & 12 & $\mathrm{y}$ \\
\hline 1998 May 06 & $08: 29$ & 87 & 293 & -20 & 141 & -13 & 141 & 1 & 6.8 & 254 & 39 & cs \\
\hline 1998 May $09^{a}$ & $03: 35$ & 134 & 264 & -22 & 139 & -14 & 138 & 5 & 7.4 & 251 & 13 & $\mathrm{nc}$ \\
\hline 1998 May 19 & $10: 27$ & 80 & 293 & 12 & 308 & 28 & 300 & 27 & 17.7 & 298 & 4 & $\mathrm{y}$ \\
\hline 1998 May 27 & $13: 45$ & 92 & 267 & 16 & 212 & 22 & 205 & 50 & 8.5 & 292 & 26 & $\mathrm{nc}$ \\
\hline 1998 Jun. 02 & 08:08 & 55 & 242 & -49 & 198 & -60 & 161 & 73 & 24.0 & 213 & 29 & $\mathrm{y}$ \\
\hline 1998 Jun. 13 & $02: 27$ & 90 & 263 & -21 & 350 & -29 & 345 & 35 & 10.0 & 240 & 22 & $\mathrm{nc}$ \\
\hline 1998 Nov. 28 & $06: 54$ & 102 & 60 & 18 & 185 & 20 & 182 & 56 & 3.3 & 65 & -5 & $\mathrm{y}$ \\
\hline 1999 Mar. 17 & $07: 26$ & 68 & 70 & 28 & 158 & 36 & 149 & 47 & 11.3 & 57 & 13 & $\mathrm{y}$ \\
\hline 1999 Mar. 18 & $03: 54$ & 65 & 63 & 28 & 158 & 36 & 149 & 47 & 11.3 & 55 & 8 & $\mathrm{y}$ \\
\hline 1999 Apr. $22^{a}$ & $02: 30$ & 66 & 241 & -16 & 220 & -23 & 219 & 9 & 7.1 & 250 & -8 & $\mathrm{nc}$ \\
\hline 1999 May 30 & $11: 06$ & 84 & 284 & -13 & 34 & -19 & 30 & 34 & 7.1 & 252 & 32 & $\mathrm{y}$ \\
\hline 1999 Jun. $02^{a, b}$ & $21: 26$ & 106 & 298 & 22 & 32 & 37 & 17 & 44 & 19.5 & 300 & 3 & $\mathrm{y}$ \\
\hline 1999 Jun. 04 ${ }^{a}$ & $09: 26$ & 52 & 270 & -13 & 34 & -19 & 30 & 34 & 7.1 & 251 & 19 & $\mathrm{y}$ \\
\hline 1999 Jun. $10^{a}$ & $14: 50$ & 69 & 77 & 25 & 96 & 36 & 85 & 45 & 14.3 & 60 & 18 & $\mathrm{y}$ \\
\hline 1999 Jun. $11^{a}$ & $11: 26$ & 103 & 63 & 32 & 77 & 45 & 75 & 8 & 12.4 & 51 & 12 & $\mathrm{y}$ \\
\hline 1999 Jun. $16^{a}$ & $04: 54$ & 77 & 60 & 27 & 20 & 37 & 5 & 56 & 16.9 & 57 & 2 & $\mathrm{y}$ \\
\hline 1999 Jul. 03 & $19: 54$ & 101 & 305 & 13 & 296 & 18 & 294 & 20 & 5.3 & 287 & -19 & $\mathrm{nc}$ \\
\hline 1999 Jul. $25^{b}$ & $13: 31$ & 100 & 313 & 35 & 35 & 43 & 29 & 40 & 9.1 & 309 & -4 & $\mathrm{y}$ \\
\hline 1999 Aug. 25 & 11:06 & 43 & 111 & -33 & 182 & -37 & 180 & 23 & 4.4 & 126 & 15 & $\mathrm{y}$ \\
\hline 1999 Oct. 13 & 09:50 & 50 & 12 & 41 & 324 & 47 & 303 & 74 & 16.2 & 24 & -12 & $\mathrm{y}$ \\
\hline $2000 \mathrm{Feb} .13^{b}$ & 22:06 & 89 & 227 & -30 & 215 & -41 & 205 & 44 & 13.4 & 233 & -6 & $\mathrm{y}$ \\
\hline 2000 Feb. 26 & $23: 54$ & 79 & 14 & 24 & 289 & 37 & 254 & 70 & 32.6 & 54 & -39 & $\mathrm{y}$ \\
\hline 2000 Mar. 02 & $13: 54$ & 77 & 231 & -18 & 332 & -25 & 330 & 20 & 7.4 & 249 & -17 & $\mathrm{y}$ \\
\hline 2000 Mar. 05 & $16: 54$ & 55 & 345 & 39 & 270 & 37 & 256 & 80 & 11.7 & 333 & -12 & $\mathrm{y}$ \\
\hline 2000 Mar. 21 & 09:06 & 60 & 322 & 33 & 104 & 44 & 72 & 71 & 27.1 & 315 & -6 & $\mathrm{y}$ \\
\hline 2000 Apr. $05^{b}$ & 00:06 & 47 & 217 & -25 & 276 & -33 & 267 & 47 & 10.9 & 242 & -25 & $\mathrm{nc}$ \\
\hline
\end{tabular}


Table 1. continued.

\begin{tabular}{|c|c|c|c|c|c|c|c|c|c|c|c|c|}
\hline \multirow[b]{2}{*}{$\begin{array}{l}\text { Date } \\
(1)\end{array}$} & \multicolumn{3}{|l|}{$\mathrm{CME}$} & \multicolumn{7}{|c|}{ SR } & \multirow[b]{2}{*}{$\begin{array}{l}\text { Deviation } \delta \\
\text { from source } \\
\quad(\mathrm{deg}) \\
(12)\end{array}$} & \multirow[b]{2}{*}{$\begin{array}{c}\text { NL-CME } \\
\text { agreement } \\
\text { (13) }\end{array}$} \\
\hline & $\begin{array}{c}\text { Time } \\
\text { (UT) } \\
(2)\end{array}$ & $\begin{array}{c}A W \\
(\mathrm{deg}) \\
(3)\end{array}$ & $\begin{array}{c}\text { Central } \\
\text { PA } \\
(\mathrm{deg}) \\
(4)\end{array}$ & \multicolumn{4}{|c|}{$\begin{array}{l}\text { Carrington coordinates } \\
(\mathrm{deg})\end{array}$} & $\begin{array}{c}\text { Tilt from } \\
\text { vertical } \gamma \\
(\mathrm{deg}) \\
(9)\end{array}$ & $\begin{array}{c}\text { Length } \\
\text { (deg) } \\
(10)\end{array}$ & $\begin{array}{c}\text { Central } \\
\text { PA } \\
(\mathrm{deg}) \\
(11)\end{array}$ & & \\
\hline 2000 Apr. $08^{b}$ & $15: 54$ & 75 & 235 & $\overline{-16}$ & 233 & -24 & 228 & 34 & 9.6 & 251 & -16 & $\mathrm{y}$ \\
\hline 2000 Apr. $23^{a}$ & $12: 54$ & 119 & 280 & 10 & 77 & 25 & 71 & 24 & 16.3 & 288 & 7 & $\mathrm{nc}$ \\
\hline 2000 May 11 & $23: 50$ & 69 & 168 & -33 & 44 & -55 & -3 & 65 & 39.3 & 146 & -22 & $\mathrm{y}$ \\
\hline 2000 May 15 & 08:50 & 84 & 59 & 20 & 301 & 26 & 298 & 23 & 6.4 & 67 & -9 & $\mathrm{nc}$ \\
\hline 2000 Jun. 02 & $20: 30$ & 115 & 77 & 22 & 80 & 17 & 80 & 0 & 4.9 & 67 & 10 & $\mathrm{nc}$ \\
\hline 2000 Jun. 25 & $07: 54$ & 55 & 268 & 12 & 265 & 17 & 253 & 67 & 12.6 & 286 & 18 & $\mathrm{y}$ \\
\hline 2000 Jun. 27 & $10: 54$ & 104 & 336 & 40 & 249 & 42 & 228 & 85 & 15.9 & 314 & -22 & $\mathrm{y}$ \\
\hline 2000 Jul. 10 & $20: 26$ & 66 & 16 & 43 & 287 & 49 & 266 & 73 & 15.8 & 44 & -27 & $\mathrm{y}$ \\
\hline 2000 Jul. 10 & $21: 50$ & 71 & 89 & 17 & 306 & 17 & 299 & 88 & 7.3 & 72 & 17 & $\mathrm{y}$ \\
\hline 2000 Jul. 17 & $08: 54$ & 120 & 94 & 3 & 240 & 6 & 231 & 74 & 9.2 & 89 & 5 & $\mathrm{y}$ \\
\hline 2000 Aug. $02^{a}$ & $17: 54$ & 88 & 42 & 37 & 330 & 53 & 310 & 53 & 20.7 & 45 & -3 & $\mathrm{y}$ \\
\hline 2000 Aug. $03^{b}$ & $08: 30$ & 78 & 301 & 16 & 120 & 31 & 106 & 44 & 19.9 & 293 & -8 & $\mathrm{y}$ \\
\hline 2000 Aug. $28^{a}$ & $00: 54$ & 86 & 63 & 25 & 346 & 40 & 315 & 64 & 30.4 & 54 & 9 & $\mathrm{y}$ \\
\hline 2000 Sep. 04 & 06:06 & 117 & 318 & 12 & 381 & 34 & 374 & 18 & 22.9 & 299 & -19 & $\mathrm{y}$ \\
\hline 2000 Sep. 12 & $11: 54$ & 181 & 204 & -13 & 251 & -24 & 234 & 56 & 19.1 & 197 & 7 & $\mathrm{y}$ \\
\hline 2000 Sep. 27 & $20: 50$ & 77 & 181 & -23 & 17 & -37 & 13 & 15 & 14.9 & 157 & -23 & $\mathrm{y}$ \\
\hline 2000 Oct. $16^{a}$ & $07: 27$ & 115 & 281 & 4 & 257 & 2 & 254 & 52 & 3.2 & 275 & -6 & $\mathrm{nc}$ \\
\hline 2000 Oct. 26 & $16: 50$ & 91 & 118 & -33 & 305 & -25 & 302 & 21 & 8.4 & 122 & 4 & $\mathrm{y}$ \\
\hline 2000 Nov. 01 & $16: 26$ & 137 & 127 & -15 & 257 & -16 & 245 & 85 & 11.6 & 117 & -10 & $\mathrm{y}$ \\
\hline 2000 Nov. 02 & $16: 26$ & 105 & 306 & 27 & 361 & 26 & 342 & 88 & 17.0 & 297 & -10 & $\mathrm{y}$ \\
\hline 2000 Nov. 04 & 01:50 & 67 & 228 & -28 & 350 & -36 & 313 & 79 & 32.1 & 235 & -7 & $\mathrm{y}$ \\
\hline 2000 Nov. $08^{b}$ & 23:06 & 126 & 293 & 19 & 251 & 25 & 249 & 25 & 6.2 & 295 & 2 & $\mathrm{y}$ \\
\hline 2000 Nov. 17 & $06: 30$ & 56 & 185 & -50 & 156 & -50 & 117 & 90 & 24.8 & 211 & -25 & $\mathrm{nc}$ \\
\hline 2000 Nov. 23 & $20: 30$ & 97 & 127 & -26 & 298 & -38 & 295 & 12 & 12.5 & 126 & -1 & $\mathrm{y}$ \\
\hline 2000 Nov. $26^{b}$ & $06: 30$ & 126 & 275 & 19 & 51 & 25 & 48 & 24 & 6.9 & 292 & 18 & $\mathrm{y}$ \\
\hline 2000 Dec. 10 & $03: 26$ & 53 & 24 & 44 & 118 & 52 & 54 & 83 & 42.6 & 33 & -9 & $\mathrm{y}$ \\
\hline 2001 Jan. 07 & 04:06 & 64 & 304 & 17 & 225 & 27 & 188 & 76 & 35.5 & 295 & -8 & $\mathrm{y}$ \\
\hline 2001 Jan. 26 & $01: 54$ & 65 & 302 & 14 & 331 & 35 & 310 & 44 & 28.7 & 297 & -5 & $\mathrm{y}$ \\
\hline 2001 Jan. 26 & $16: 06$ & 98 & 57 & 20 & 186 & 31 & 173 & 50 & 16.7 & 59 & -2 & $\mathrm{y}$ \\
\hline 2001 Feb. 02 & $19: 54$ & 85 & 62 & 17 & 97 & 22 & 93 & 38 & 6.9 & 61 & 1 & $\mathrm{y}$ \\
\hline 2001 Feb. 20 & $15: 05$ & 70 & 51 & 18 & 186 & 25 & 177 & 51 & 11.0 & 69 & -18 & $\mathrm{y}$ \\
\hline 2001 Feb. $26^{b}$ & $18: 50$ & 86 & 242 & -23 & 278 & -40 & 260 & 47 & 22.8 & 239 & 3 & $\mathrm{y}$ \\
\hline 2001 Apr. $01^{a}$ & $11: 26$ & 98 & 105 & -20 & 360 & -27 & 357 & 20 & 8.3 & 116 & 11 & $\mathrm{y}$ \\
\hline 2001 Apr. 03 & $03: 26$ & 95 & 109 & -18 & 360 & -26 & 357 & 20 & 8.3 & 111 & 2 & $\mathrm{y}$ \\
\hline 2001 Apr. 09 & 00:06 & 74 & 131 & -24 & 360 & -27 & 356 & 52 & 5.2 & 159 & 27 & $\mathrm{y}$ \\
\hline 2001 Apr. 15 & $14: 06$ & 94 & 271 & -25 & 360 & -20 & 358 & 29 & 5.1 & 248 & 23 & $\mathrm{y}$ \\
\hline 2001 Apr. $23^{b}$ & 19:09 & 69 & 236 & -30 & 249 & -36 & 232 & 71 & 15.5 & 236 & 0 & $\mathrm{y}$ \\
\hline 2001 May $15^{a}$ & $18: 52$ & 68 & 56 & 15 & 152 & 34 & 129 & 51 & 29.0 & 65 & -10 & $\mathrm{y}$ \\
\hline 2001 May 25 & $17: 26$ & 59 & 93 & -7 & 36 & -21 & 22 & 45 & 19.6 & 104 & 11 & $\mathrm{nc}$ \\
\hline 2001 Jun. $26^{a}$ & $12: 30$ & 83 & 107 & -8 & 317 & -17 & 316 & 5 & 8.5 & 102 & -5 & $\mathrm{y}$ \\
\hline $2001 \mathrm{Jul} .12^{b}$ & 00:06 & 110 & 231 & -28 & 272 & -43 & 259 & 41 & 18.3 & 230 & 0 & $\mathrm{y}$ \\
\hline $2001 \mathrm{Jul} .23^{a}$ & 11:08 & 68 & 291 & 5 & 142 & 7 & 142 & 0 & 1.9 & 276 & -15 & $\mathrm{y}$ \\
\hline 2001 Aug. $09^{a}$ & $20: 30$ & 67 & 118 & -10 & 89 & -30 & 65 & 50 & 29.7 & 110 & -9 & $\mathrm{y}$ \\
\hline 2001 Aug. 19 & 06:06 & 74 & 285 & 27 & 134 & 28 & 126 & 79 & 7.3 & 297 & 12 & $\mathrm{y}$ \\
\hline 2001 Aug. $21^{b}$ & $12: 06$ & 104 & 231 & -19 & 80 & -25 & 68 & 64 & 12.7 & 237 & -6 & $\mathrm{y}$ \\
\hline 2001 Aug. 24 & 09:26 & 61 & 121 & -21 & 290 & -15 & 287 & 27 & 7.1 & 114 & -7 & $\mathrm{cs}$ \\
\hline
\end{tabular}




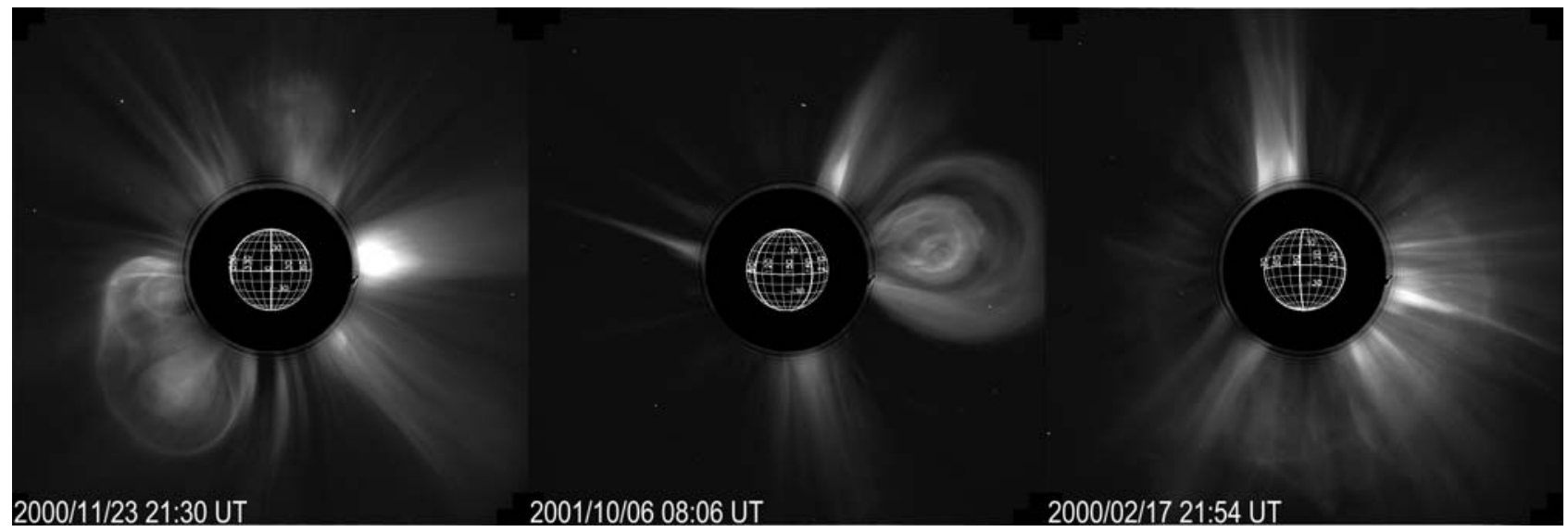

Fig. 1. Two structured CME events (left and centre) and an unstructured one, in this case a halo CME, as observed by the LASCO C2 coronagraph on board SOHO.

Table 1. continued.

\begin{tabular}{|c|c|c|c|c|c|c|c|c|c|c|c|c|}
\hline \multirow[b]{3}{*}{$\begin{array}{l}\text { Date } \\
\text { (1) }\end{array}$} & \multicolumn{3}{|l|}{$\overline{\mathrm{CME}}$} & \multicolumn{7}{|c|}{$\overline{\mathrm{SR}}$} & \multirow{3}{*}{$\begin{array}{l}\text { Deviation } \delta \\
\text { from source } \\
\quad(\mathrm{deg}) \\
(12)\end{array}$} & \multirow{3}{*}{$\begin{array}{l}\text { NL-CME } \\
\text { agreement* } \\
\quad(13)\end{array}$} \\
\hline & \multirow{2}{*}{$\begin{array}{c}\text { Time } \\
(\mathrm{UT}) \\
(2)\end{array}$} & \multirow{2}{*}{$\begin{array}{c}A W \\
(\mathrm{deg}) \\
(3)\end{array}$} & \multirow{2}{*}{$\begin{array}{c}\text { Central } \\
\text { PA } \\
(\mathrm{deg}) \\
(4)\end{array}$} & \multicolumn{4}{|c|}{$\begin{array}{c}\text { Carrington coordinates } \\
\text { (deg) }\end{array}$} & \multirow{2}{*}{$\begin{array}{c}\text { Tilt from } \\
\text { vertical } \gamma \\
\text { (deg) } \\
\text { (9) }\end{array}$} & \multirow{2}{*}{$\begin{array}{c}\text { Length } \\
\text { (deg) } \\
\text { (10) }\end{array}$} & \multirow{2}{*}{$\begin{array}{c}\text { Central } \\
\text { PA } \\
(\mathrm{deg}) \\
(11)\end{array}$} & & \\
\hline & & & & $\begin{array}{l}\theta_{1} \\
(5)\end{array}$ & $\begin{array}{l}\phi_{\mathrm{C} 1} \\
(6)\end{array}$ & $\begin{array}{l}\theta_{2} \\
(7)\end{array}$ & $\begin{array}{l}\phi_{\mathrm{C} 2} \\
(8)\end{array}$ & & & & & \\
\hline 2001 Sep. 21 & $08: 54$ & 63 & 117 & -15 & 276 & -22 & 272 & 28 & 7.7 & 113 & -4 & $\mathrm{cs}$ \\
\hline 2001 Sep. 28 & $10: 30$ & 91 & 195 & -15 & 277 & -22 & 270 & 45 & 9.9 & 226 & -31 & $\mathrm{nc}$ \\
\hline 2001 Oct. 01 & 05:30 & 106 & 237 & -19 & 292 & -21 & 282 & 77 & 9.3 & 248 & -11 & $\mathrm{y}$ \\
\hline 2001 Oct. 06 & $05: 54$ & 66 & 276 & 13 & 229 & 11 & 228 & 31 & 2.7 & 282 & 5 & cs \\
\hline 2001 Oct. 29 & $11: 50$ & 72 & 296 & 14 & 285 & 34 & 262 & 49 & 29.0 & 294 & -2 & $\mathrm{y}$ \\
\hline 2001 Nov. 01 & $14: 30$ & 90 & 127 & -18 & 80 & -22 & 80 & 9 & 4.3 & 111 & -16 & $\mathrm{y}$ \\
\hline 2001 Dec. $02^{a}$ & $22: 06$ & 92 & 263 & -8 & 193 & -11 & 193 & 7 & 2.5 & 261 & 2 & cs \\
\hline 2001 Dec. $14^{a}$ & 09:06 & 107 & 72 & 2 & 219 & 9 & 214 & 38 & 8.2 & 85 & -13 & $\mathrm{nc}$ \\
\hline 2001 Dec. 20 & $00: 30$ & 69 & 114 & -17 & 208 & -26 & 195 & 57 & 15.5 & 122 & 8 & $\mathrm{y}$ \\
\hline 2001 Dec. $28^{a}$ & $20: 06$ & 108 & 115 & -17 & 20 & -28 & 16 & 25 & 11.2 & 114 & -1 & $\mathrm{y}$ \\
\hline 2002 Jan. 04 & 09:30 & 90 & 46 & 19 & 338 & 32 & 314 & 62 & 25.5 & 62 & -16 & $\mathrm{y}$ \\
\hline 2002 Jan. $24^{a}$ & $03: 54$ & 94 & 99 & -20 & 20 & -28 & 11 & 49 & 11.3 & 119 & 20 & $\mathrm{nc}$ \\
\hline 2002 Feb. 12 & $15: 30$ & 115 & 67 & 10 & 206 & 13 & 202 & 52 & 4.9 & 63 & 4 & $\mathrm{nc}$ \\
\hline 2002 Feb. $28^{a, b}$ & $00: 30$ & 97 & 252 & -17 & 143 & -28 & 136 & 34 & 12.2 & 246 & 6 & $\mathrm{y}$ \\
\hline 2002 Mar. 02 & $15: 06$ & 60 & 112 & -13 & 290 & -35 & 257 & 56 & 36.9 & 114 & 3 & $\mathrm{y}$ \\
\hline 2002 Mar. 13 & $23: 54$ & 59 & 117 & -19 & 143 & -25 & 135 & 56 & 9.5 & 111 & -6 & cs \\
\hline 2002 Mar. 18 & $02: 54$ & 166 & 256 & -16 & 180 & -2 & 177 & 12 & 14.3 & 262 & -6 & $\mathrm{y}$ \\
\hline 2002 Apr. $18^{b}$ & $16: 06$ & 69 & 254 & -10 & 190 & -21 & 185 & 24 & 12.0 & 255 & 0 & $\mathrm{y}$ \\
\hline 2002 Apr. $21^{b}$ & $01: 27$ & 117 & 273 & -13 & 154 & -28 & 132 & 56 & 25.5 & 250 & 22 & $\mathrm{y}$ \\
\hline 2002 May 21 & $21: 50$ & 105 & 53 & 18 & 346 & 23 & 342 & 37 & 6.2 & 57 & -5 & $\mathrm{y}$ \\
\hline 2002 May $22^{b}$ & 00:06 & 87 & 243 & -14 & 92 & -19 & 87 & 48 & 7.8 & 253 & -10 & $\mathrm{y}$ \\
\hline 2002 May 22 & $03: 50$ & 145 & 237 & 1 & 84 & -29 & 60 & 38 & 38.0 & 254 & -16 & $\mathrm{y}$ \\
\hline 2002 Aug. 01 & 04:06 & 118 & 201 & -16 & 142 & -28 & 141 & 7 & 11.6 & 150 & -51 & $\mathrm{y}$ \\
\hline 2002 Aug. 13 & 08:54 & 104 & 46 & 38 & 299 & 42 & 285 & 75 & 11.6 & 49 & -3 & $\mathrm{y}$ \\
\hline 2002 Aug. 23 & 08:50 & 78 & 84 & -5 & 157 & -10 & 154 & 25 & 5.3 & 100 & 16 & $\mathrm{y}$ \\
\hline 2002 Oct. 13 & $23: 54$ & 112 & 91 & -10 & 194 & -20 & 186 & 40 & 12.9 & 107 & 16 & $\mathrm{y}$ \\
\hline 2002 Oct. 15 & $12: 54$ & 82 & 187 & -25 & 252 & -25 & 198 & 90 & 48.5 & 154 & -33 & $\mathrm{y}$ \\
\hline 2002 Oct. 25 & $07: 27$ & 51 & 121 & -30 & 71 & -40 & 35 & 75 & 30.9 & 132 & 11 & $\mathrm{y}$ \\
\hline 2002 Nov. 19 & 03:06 & 89 & 20 & 28 & 119 & 34 & 113 & 44 & 7.8 & 42 & -22 & $\mathrm{nc}$ \\
\hline 2002 Nov. 26 & $17: 06$ & 77 & 300 & 22 & 129 & 26 & 126 & 30 & 4.2 & 294 & -6 & $\mathrm{y}$ \\
\hline 2002 Dec. $28^{a}$ & $16: 30$ & 72 & 308 & 27 & 109 & 35 & 83 & 72 & 24.0 & 303 & -5 & nc \\
\hline
\end{tabular}

${ }^{*} \mathrm{y}=$ agreement, $\mathrm{nc}=$ not clear agreement, $\mathrm{cs}=$ compact system.

${ }^{a}$ Far-side events, i.e. with mean absolute heliographic longitude greater than $90^{\circ}$.

${ }^{b}$ Events with neutral lines parallel to the limb, taken into account for Fig. 17. 


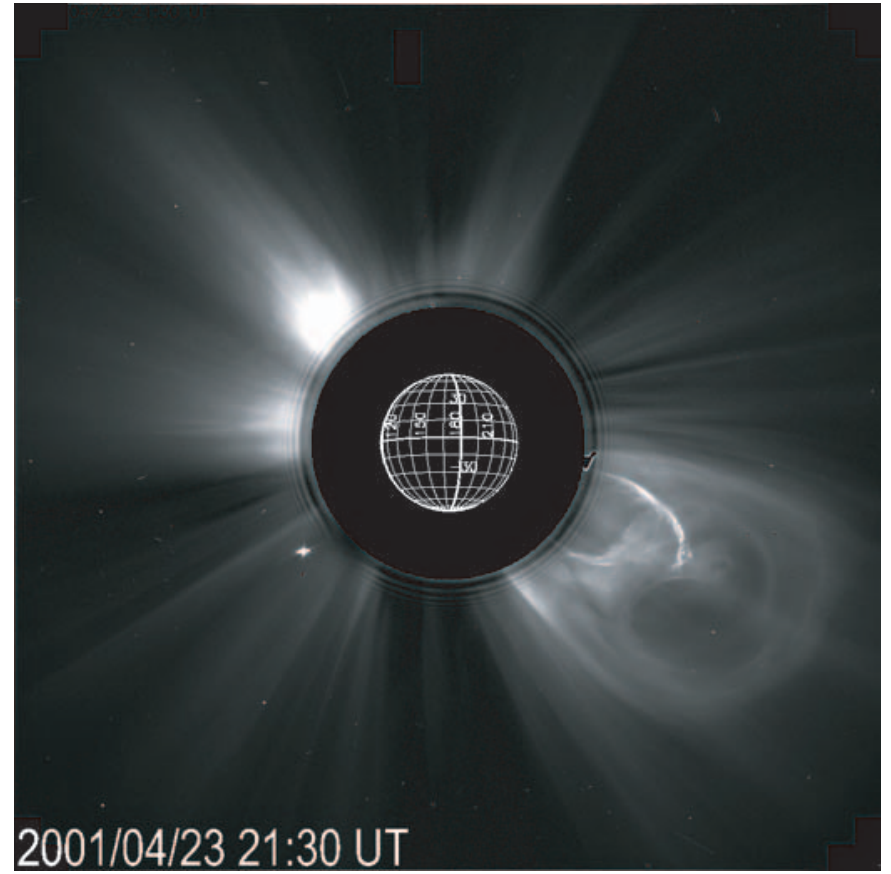

Fig. 2. Structured CME event observed on 23rd April 2001 by LASCO/C2, with a circular dark cavity that can be interpreted as a magnetic flux rope.

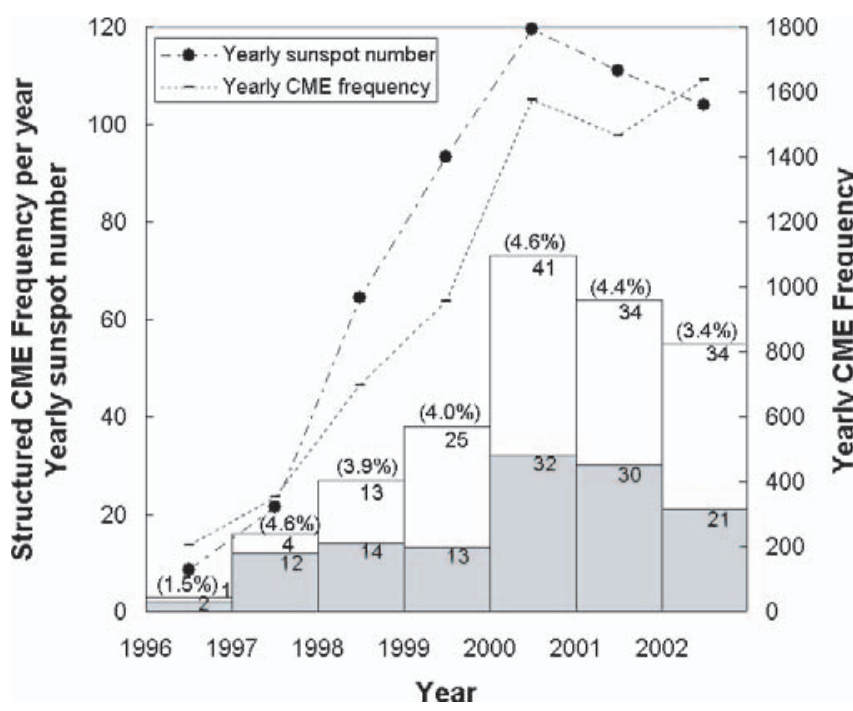

Fig. 3. Time distribution histogram for the 276 selected structured CMEs (columns) in comparison with the yearly sunspot number (dash-dotted line with circles) and the total CME frequency per year as taken from the CME Catalog (dotted line with horizontal ticks). Gray-shaded portions of the columns represent the number of events for which the source region could be identified. The numbers in brackets indicate the fraction of structured events with respect to the total CME frequency per year.

Although included in the CME Catalog, the central position angle (PA) and angular width $(A W)$ of the CMEs were recalculated, because the method used to determine the values provided by the Catalog takes into account brightness differences with respect to the surrounding corona, which often were found not to be part of the structured CME itself, but rather

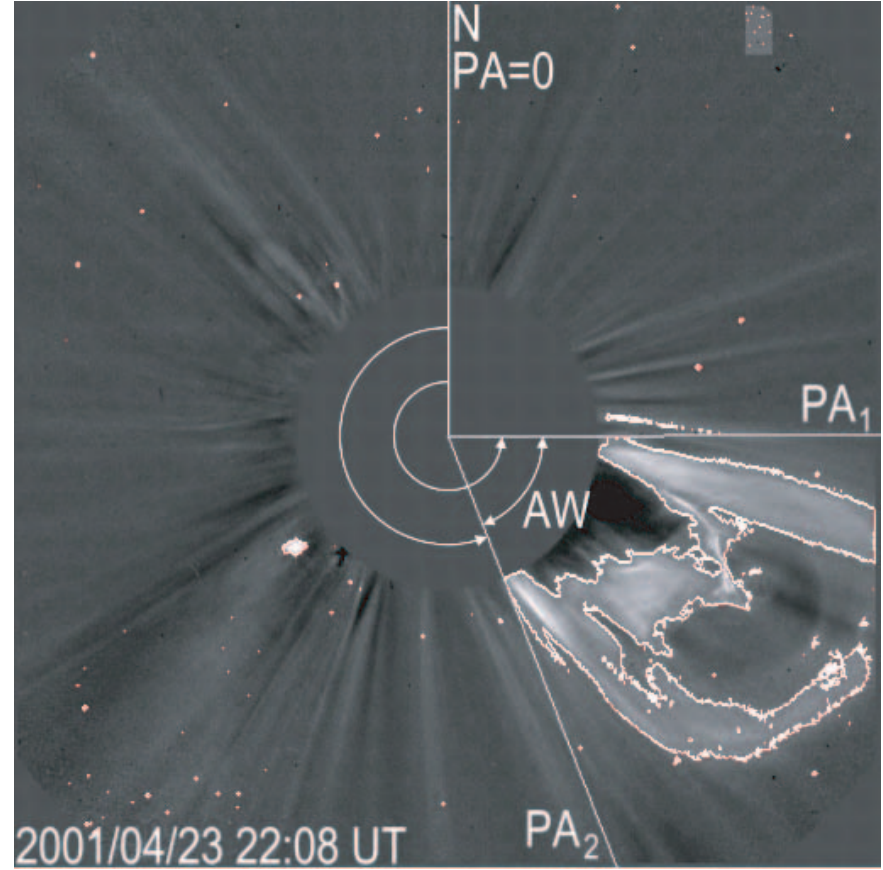

Fig. 4. LASCO $C 2$ image of a structured CME, from which a pre-event image taken at 18:11 UT has been subtracted. A white contour delineates the structured CME, which is delimited by the position angles of its two outer edges (PA1 and PA2), subtending an angular width $A W$.

deflections of pre-existing coronal structures or short-lived wave-like disturbances of the ambient corona, as addressed by Michalek et al. (2003).

To recalculate these two angular parameters for the selected CMEs we used LASCO C2 differential images, specifically the last $\mathrm{C} 2$ image in time that showed the leading edge of the $\mathrm{CME}$ before it left the FOV of C2, as shown in Fig. 4. With the aid of brightness contour levels, taking into account the brightness of the individual event and that of the background corona, the $A W$ and central PA were calculated as $A W=\mathrm{PA}_{2}-\mathrm{PA}_{1}$ and $\mathrm{PA}_{\text {central }}=\left(\mathrm{PA}_{1}+\mathrm{PA}_{2}\right) / 2$. Where $\mathrm{PA}_{1}$ and $\mathrm{PA}_{2}$ represent the two outer edges of the CME projected in the plane of the sky measured counter clockwise from the solar north $\left(\mathrm{PA}=0^{\circ}\right)$, with vertex in the centre of the solar disk.

The recalculated values are given in Table 1 and can be compared to those of the CME Catalog. The individual differences range from just a few to more than two hundred degrees. The frequency distribution of the recalculated $A W \mathrm{~s}$ is presented in Fig. 5 as a histogram with bins of $15^{\circ}$. The maximum frequency lies in the range $75^{\circ}$ to $90^{\circ}$, with an average (projected) $A W$ of $85^{\circ}$, whereas the average $A W$ as calculated from the CME Catalog would be $155^{\circ}$, since the method used to determine the latter values includes also disturbances sometimes not part of the CME, yielding therefore some doubtful halos. The finding implies that CME parameters need to be treated carefully in general, especially when physical quantities, such as CME masses, are considered.

When compared with the mean $A W \mathrm{~s}$ of previously observed CMEs, it is worth to point out that the average $A W$ of structured CMEs is substantially larger. For instance, the average $A W$ obtained for the CMEs detected by the Solwind 


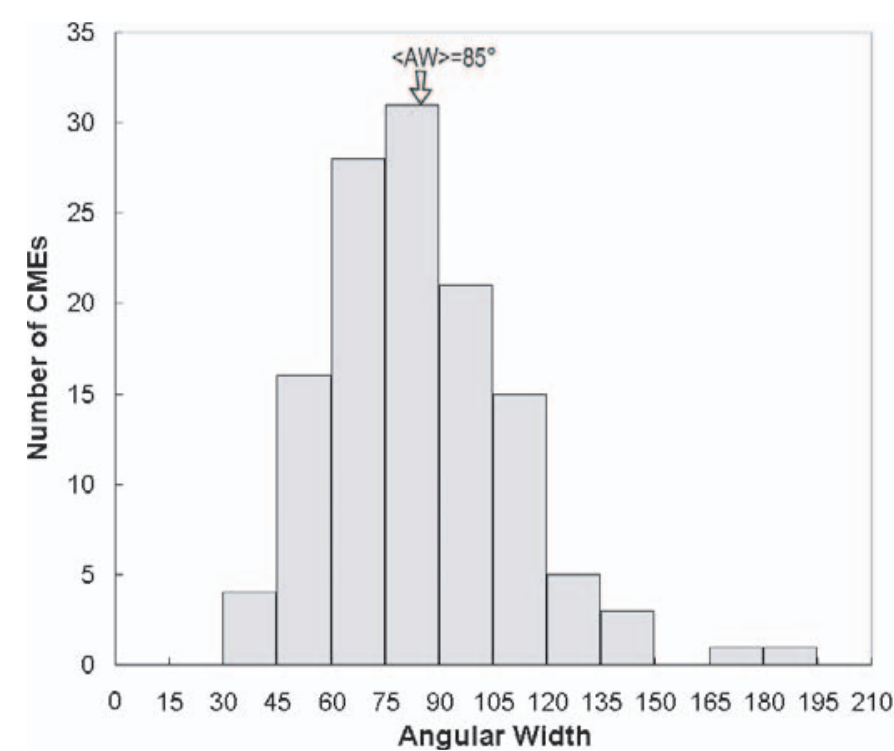

Fig. 5. Angular width distribution histogram for the 124 structured CMEs with identified source regions. The arrow indicates the average $A W$.

instrument onboard the P-78 spacecraft during 1979-1981 was $45^{\circ}$, and $24^{\circ}$ for the years 1984-1985 (Howard et al. 1985, $1986)$. For the 1300 CMEs recorded by the SMM instrument during 1980 and 1984-1989, the average $A W$ resulted to be $47^{\circ}$ (Hundhausen 1993), while the mean value of the $A W$ for the 240 CMEs recorded by the ground-based coronagraph MK3 during 1980-1989 yielded 37 (St. Cyr et al. 1999). Furthermore, St. Cyr et al. (2000) determined an average $A W$ of $72^{\circ}$ for the 841 LASCO CMEs identified between January 1996 and June 1998; whereas Yashiro et al. (2003) calculated yearly averaged $A W \mathrm{~s}$ for the LASCO CMEs during the period 1996-2001, yielding values ranging from $51^{\circ}$ up to $66^{\circ}$. The larger value of the average $A W$ obtained for structured CMEs seems to be closely related to the selection criterion: the most spectacular events were all quite sizeable, whereas narrow CMEs typically showed no structure. Note that the overall shape of the distribution displayed in Fig. 5 agrees with that of previous studies (Howard et al. 1985, 1986; Hundhausen 1993; St. Cyr et al. 1999; Gopalswamy et al. 2003), somewhat skewed on the right, but displaced toward higher values.

Based on the recalculated $A W \mathrm{~s}$, new PAs were determined, listed in Col. 4 of Table 1. The characteristics of the PAs are discussed together with those of the corresponding source regions in Sect. 5 (see Fig. 18).

\section{Source regions}

In order to identify the source region (SR) in the low corona for each of the 276 structured CMEs, EIT observations taken at $195 \AA$ were searched for pre- and post-eruptive signatures of CMEs, like: outward motion of coronal material in form of loops or eruptive prominences (e.g. Webb \& Hundhausen 1987), flares (Hudson et al. 1998), coronal waves and dimmings (e.g. Thompson et al. 1998) or post-eruptive loops/arcades (Sterling et al. 2000; Tripathi et al. 2004).

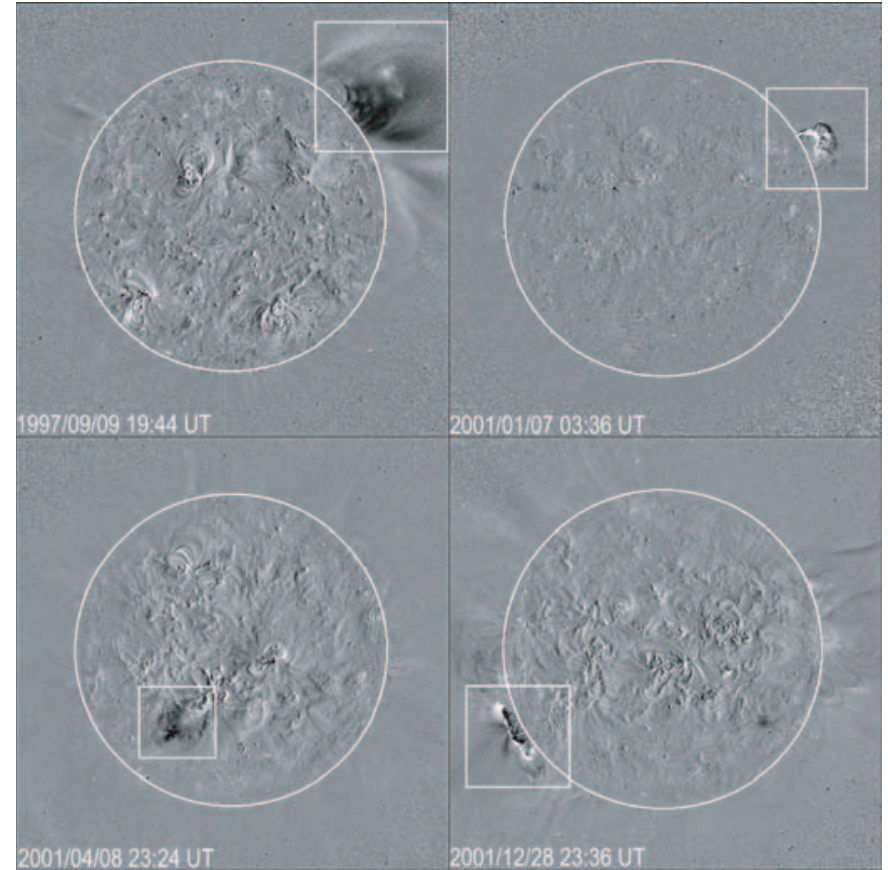

Fig. 6. Examples of eruptive (top) and post-eruptive (bottom) signatures associated with four structured CMEs. Features within the squares, in clockwise direction: Rising low coronal loops, a prominence eruption, a post-eruptive arcade and a coronal dimming. Note that the latter one is associated with the CME first detected by $\mathrm{C} 2$ on 9th April 2001, at 00:06 UT.

Figure 6 shows in the clockwise direction as an example the signatures that were associated with four of the CME events of Table 1: rising low coronal loops, a prominence eruption, a post-eruptive arcade and a coronal dimming. For each event the time and spatial relationship of LASCO and EIT features was investigated, in order to make proper associations, especially difficult at times of high solar activity. The time of the first observation of the CME in the FOV of $\mathrm{C} 2$ needed to be in agreement with the expected propagation time of the features seen by EIT lower down in the corona, as verified with the aid of height-time diagrams taken from the CME Catalog. Of course, the features identified in EIT observations needed to be enveloped by the two edge PAs defining the CME spatial extent. Additionally $\mathrm{H} \alpha$ and SXT data from Yohkoh were consulted in order to ensure the veracity of the candidate SR.

As already mentioned in Sect. 3, the SR could be identified for $124(45 \%)$ events out of the total number of 276 structured CMEs (see Table 1). Figure 7 indicates the frequency distribution of the relative location of the SRs on the Sun. As limb cases we considered all those events that were found to be not further away from the limb than $\pm 20^{\circ}$ in heliographic longitude, i.e. events in the range $70^{\circ}$ to $110^{\circ}$ in longitude with respect to central meridian. Events between $50^{\circ}$ and $70^{\circ}$ heliographic longitude were classified as near limb events. Finally, the category disk events was devoted to those SRs located at the remaining longitudes. Some far-side SRs could be identified, especially at times of low activity, when the position angle of the CME matched the location of well-pronounced isolated regions that were seen in the magnetogram before they entered the far side 


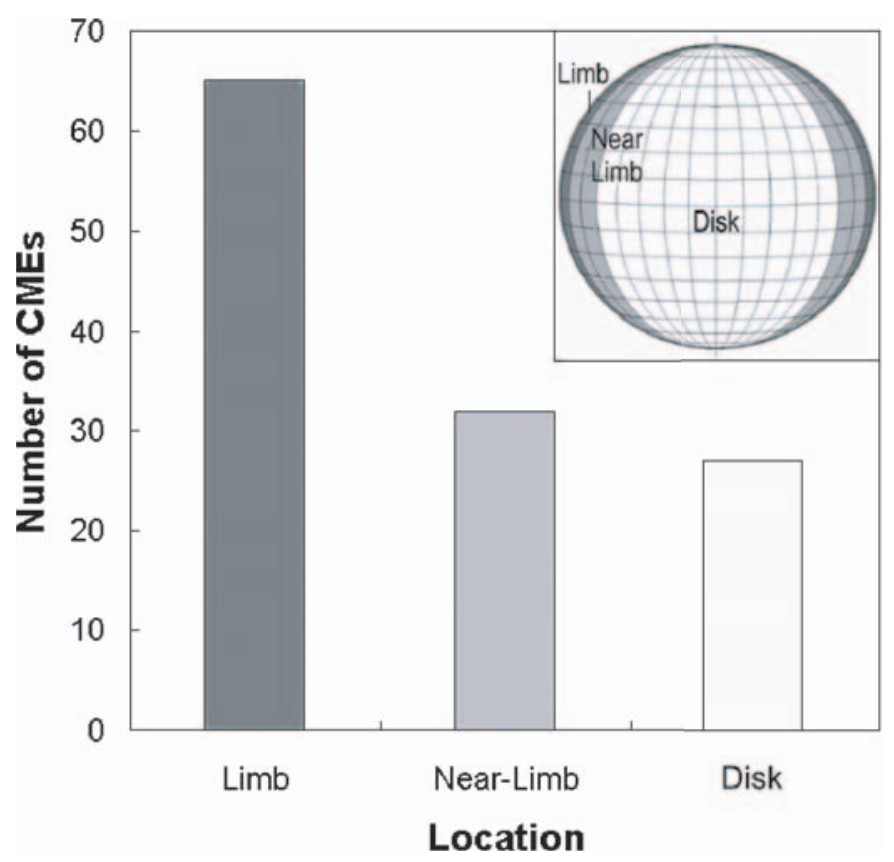

Fig. 7. Distribution of the identified SRs on the Sun, as seen from SOHO. The inset explains the bin tags: Limb cases classify those events with SRs located not further away from the limb than $\pm 20^{\circ}$ in heliographic longitude $\left(70^{\circ}\right.$ to $110^{\circ}$ from central meridian), Near Limb refers to those events with SRs between $50^{\circ}$ and $70^{\circ}$ heliographic longitude, and the category Disk comprises the events with SRs situated at the remaining longitudes.

of the sun or when they appeared at the east limb, with noticeable eruptive and post-eruptive signatures from SRs that were just behind the limb. Such cases, i.e. those whose mean absolute heliographic longitudes were greater than $90^{\circ}$, have been denoted by a superscript $a$ in Table 1 . The least likely place that allows to determine the SR of a CME lies close to disk centre on the opposite side of the sun, as viewed from SOHO. For 152 events (55\%) the SR could not be identified, most often because they were far sided and/or they showed very weak or too complex signatures. As can be seen from Fig. 7, and as may be anticipated from the definition of structured CMEs, the highest percentage of events resulted from SRs located at and near the limb. This fact indicates that projection effects indeed play a major role in the appearance of CMEs in the FOV of the LASCO coronagraphs so that halo CMEs may not be different from structured CMEs in general. Since limb events provide the purest samples to determine the physical parameters characterizing CMEs, such as $A W$, speed or mass, the identified set of structured events provides a unique database. Limb CMEs keep their $A W$ during their outward propagation, while CMEs originating in other regions of the solar disk will gain $A W$ as they travel farther away from the Sun due to projection effects, e.g. about half of all halos turn into full halos shortly after they are detected, and from then on encompass completely the occulting disk. Further, the observed speed of the leading edge on the plane of the sky of limb CMEs is approximately equal to the real propagation speed of the CME. In order to locate the photospheric counterparts of the 124 found SRs, MDI synoptic charts were employed. As shown in Fig. 8, where the SRs for the CMEs selected during Carrington rotation 1961 (see Table 1) are denoted by dashed circles, the SRs were generally related with opposite polarity regions in both hemispheres, in agreement with the findings of Subramanian \& Dere (2001). Note that the term opposite polarity regions is used because it comprises not only active bipolar regions but also decaying ones, and hence it can be employed for bipolar regions independently of their characteristics. The solid line within the circles defines the disturbed portion of the polarity inversion line, identified through the position of the filament that erupted or through the location of post-eruptive EUV arcades or dimmings. The Carrington coordinates of the disturbed portion of the polarity inversion line, given by $\theta_{1}, \phi_{\mathrm{C} 1}, \theta_{2}$, and $\phi_{\mathrm{C} 2}$ in Cols. 5 to 8 of Table 1, were inferred directly from $\mathrm{H} \alpha$ images in cases of clear filament disappearances and from EIT images if clear post-eruptive loops were observed. In the other cases the coordinates resulted from a combined investigation of EIT, $\mathrm{H} \alpha$ and MDI data to minimize uncertainties in the heliographic coordinates.

Figure 9 illustrates the position and length of the SRs of the structured CMEs. During the years 1996-2002 a big concentration of SRs is found in the two activity region belts centred at $\pm 20^{\circ}$ latitude with respect to the solar Equator, whereas at transequatorial latitudes and at latitudes above $60^{\circ}$ no SRs could be observed. The typical orientation of the SRs in the two hemispheres, being NE to SW in the N and NW to SE in the $S$, is following Joy's Law (Hale et al. 1919), according to which the following polarity of sunspot pairs tends to appear farther away from the Equator than that of the preceding one of opposite polarity. Figure 10a displays the tilt angle $\gamma(\mathrm{Col} .9$ in Table 1) of the SRs as a function of time between 1996 and 2002. The angle $\gamma$ is measured with respect to the north-south line (Trottet \& MacQueen 1980), ranging from $0^{\circ}$ to $90^{\circ}$ with a tilt of $0^{\circ}$ corresponding to a vertical neutral line and one of $90^{\circ}$ corresponding to a completely horizontal line. The evolution of $\gamma$ in time until shortly after solar maximum reveals no clear systematic trend, but a tendency for lower inclined cases $(\gamma$ greater than $45^{\circ}$ ) is observed after mid 2000 , i.e. after the Sun's global magnetic polarity reversal, as indicated by the averages representing sets of ten consecutive values. The comparison of Fig. 10a with the variation in time of the SR latitudes shown in Fig. 10b yields a similar behaviour, meaning that higher latitude SRs are more horizontally inclined (high $\gamma$ ) while lower latitude ones are more vertically inclined (small $\gamma$ ), as can also be seen in Fig. 9. The variation of $\gamma$ with respect to the SR latitude for both hemispheres during 1996-2002 is displayed in Fig. 11. A linear fit yields $\gamma=1.09 \theta+20$ for the northern and $\gamma=-0.96 \theta+16.6$ for the southern hemisphere. The latitudinal dependence of $\gamma$ is more pronounced in the northern hemisphere related to a different latitudinal distribution of the SRs in both hemispheres. The average SR latitude in the $\mathrm{N}$ was $25^{\circ}$ and $-24^{\circ}$ in the $\mathrm{S}$.

The appearance of higher latitude SRs during the first half of 1998 and second half of 2000 seems to be consistent with times of large spread in latitude of newly emerging magnetic flux and strong flux migrations towards the poles (e.g. Benevolenskaya et al. 2002). 

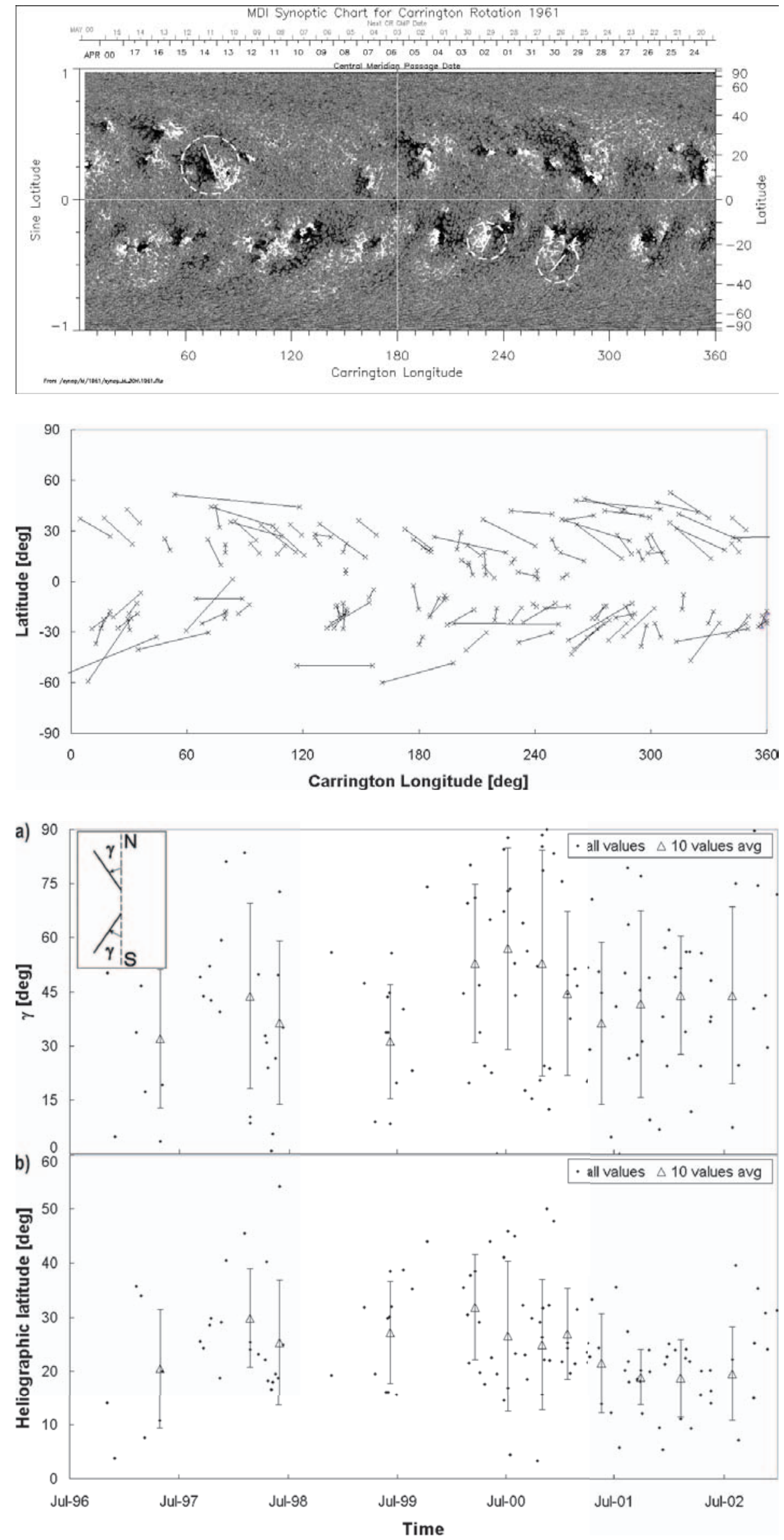

Fig. 8. SOHO MDI synoptic chart exhibiting (encircled) the photospheric source regions of three structured CMEs as identified from backtracking of EIT images. The straight line symbolises the neutral line separating opposite polarities. White represents a positive magnetic polarity(field lines pointing away form the Sun) and black a negative one (field lines pointing towards the Sun).

Fig. 9. The positions and lengths of the structured CME SRs identified during 1996-2002, represented by lines, and compiled in a Carrington Map.

Fig. 10. a) Evolution of the structured CME SR tilt angle $\gamma$ in time. The direction in which $\gamma$ grows is sketched in the inset. A vertical neutral line is represented by $\gamma=0^{\circ}$, a horizontal one by $\gamma=90^{\circ}$. b) Absolute SR latitude variation in time. The error bars are given by the standard deviation of the corresponding averaged points. 


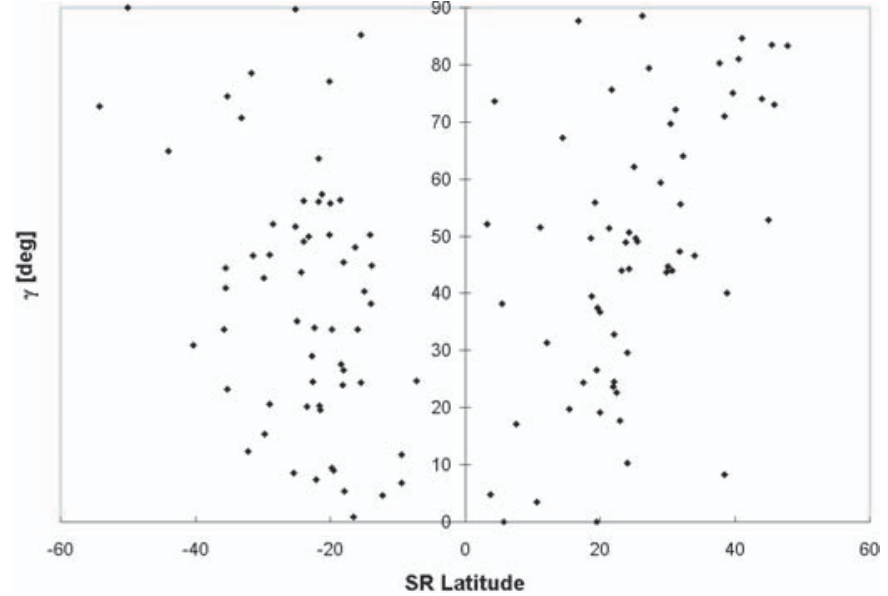

Fig. 11. Tilt angle $\gamma$ of the structured CME SRs as a function of heliographic latitude with negative values representing southern latitudes. $\gamma=0^{\circ}$ represents vertically oriented SRs.

The observed lengths of the SRs (Col. 10 in Table 1) ranged from a few degrees up to more than $40^{\circ}$, as can be seen in the histogram displayed in Fig. 12, showing a poissonian-type distribution with its maximum located in the range of $6^{\circ}-12^{\circ}$ and average length of $14^{\circ}$. The lengths of the SRs are considerably smaller than the $A W \mathrm{~s}$ of the respective CMEs (see Table 1), indicating an enormous expansion of magnetic structures from the low corona into the FOV of C2 (to compare with Figs. 13 and 14).

\section{Comparison of CME features and SR properties}

Each structured CME-SR pair was investigated for similarities between magnetic structures apparent in the low corona at EUV wavelengths and in white-light in the FOV of LASCO C2. In Fig. 13 the evolution of the structured CME on January 4, 2002 can be followed beginning with the rise of the prominence and surrounding loops at around 09:12 UT up to its development in $\mathrm{C} 2$. In the left frame of Fig. 13a the prominence is observed to lie almost perpendicular to the line of the limb, i.e., being perpendicular to the line of sight. This prominence also appears to be underneath a loop system which grows in size as the prominence rises and lifts off (mid and right frames of Fig. 13a. This structured CME can be considered as a typical three-part CME where the dark cavity, embedded by the bright leading edge and trailing prominence material, is commonly interpreted as the site of a magnetic flux rope (e.g. Chen et al. 1997; Dere et al. 1999; Hundhausen 1999; Wood et al. 1999; Gibson \& Low 2000).

The existence of large-scale expanding helical flux ropes is supported by satellite observations of magnetic clouds in the solar wind and their association with disappearing filaments (e.g. Bothmer \& Schwenn 1998) and also by theoretical models (e.g. Low 2001). The authors assume that the prominence is likely representing the bottom of a large scale flux rope above it. In some CMEs detected by LASCO the flux rope structure seems to be directly visible, such as for the large CME that originated near the SW limb on June 2, 1998 in association

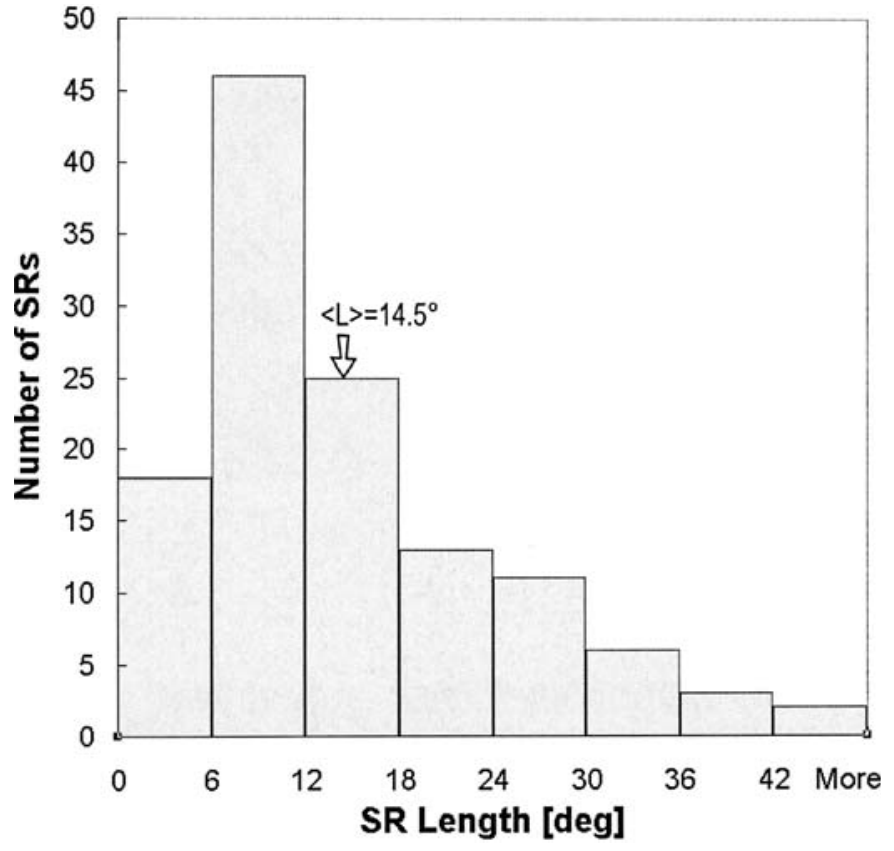

Fig. 12. Distribution histogram of the SR lengths in bins of 6 degrees. The average SR length is indicated by the arrow.

with a prominence eruption (Plunkett et al. 2000; Srivastava et al. 2000).

In Fig. 13b, the generic structure of the core material of the $\mathrm{CME}$ later seen in $\mathrm{C} 2$ matches the structure of the erupting prominence in EIT and the growing loops above the prominence correspond with what later is referred to as the leading edge of the CME (Fig. 13b). A deeper look at the source region, by superposing MDI and EIT images from two days after the event when the CME's SR appeared on the visible disk (Fig. 13c), shows that the filament originated from a filament channel that lay along the neutral line separating opposite polarities. The whole system was oriented almost perpendicular to the line of the limb.

In contrast, when the neutral line is approximately parallel to the line of the limb as indicated by the post eruptive arcade in Fig. 14a and by the neutral line evident in Fig. 14c, the shape of the subsequent CME is essentially different (Fig. 14b), being a linear feature parallel to the limb and having a broad, diffuse inner core. The generic structure of this CME in C2, apparent as a side-viewed prominence/overlying loop system, can be identified already in the EIT images, supporting the assumption of self-similar expanding structures.

The two structured CMEs shown in Figs. 13 and 14 exhibit archetypical features representative for most of the events collected in Table 1. These basic characteristics can be interpreted through expanding loop systems with cylindrical symmetry agreeing with the magnetic flux rope nature evident in some events (see Fig. 2). Based on this interpretation, the two complementary cases of Figs. 13 and 14 show a CME from two very different perspectives. In the case of Fig. 13, the CME is observed along its symmetry axis, while in the event of Fig. 14 the line of sight is perpendicular to the symmetry axis. Assuming that the typical orientation of the neutral lines 

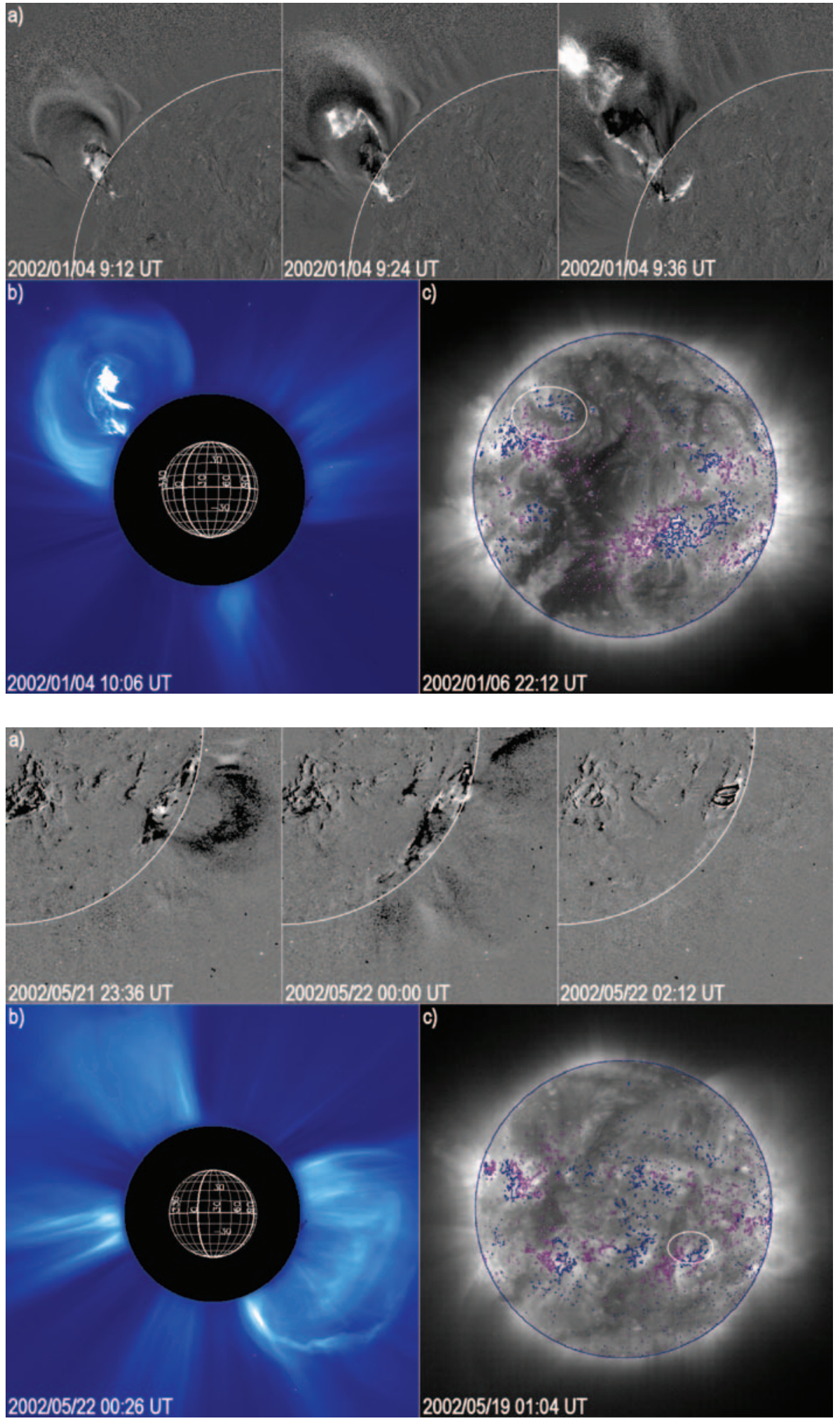

Fig. 13. Structure and evolution of the CME seen on 4th Jan. 2002. a) Left to right: EIT running difference images of the prominence eruption. b) The resulting CME in LASCO $\mathrm{C} 2$. The FOV has been reduced to focus on the CME. c) Contours of an MDI magnetogram superposed to the EIT image of the low corona at $195 \AA$, from observation taken two days later when the SR (encircled) became visible on the disk. The magenta contours represent a positive polarity, while the blue contours represent a negative one.
Fig. 14. Structure and evolution of the CME seen on 22nd May 2002. a) Left to right: EIT running difference images of the rising loops. b) The resulting CME in LASCO C2. The FOV has been reduced to focus on the CME. c) Contours of an MDI magnetogram superposed to the EIT image of the low corona at $195 \AA$, from observations taken three days before when the SR (encircled) was visible on the disk. The magenta contours represent a positive polarity, while the blue contours represent a negative one. follows Joy's Law (see Sect. 4), for front-side cases close to the east limb, the neutral lines would be approximately perpendicular to the limb, whereas for cases near the west limb the neutral lines would be roughly parallel to the line of the limb. This implies that front-sided CMEs originating from near the east limb would be prone to be seen along their symmetry axis, while those ones from near the west limb would tend to have their axis oriented perpendicular to the line of sight, as sketched in Fig. 15a. The examples selected out of Table 1 show perfect cases of these two types of projected CMEs in Fig. 15b. East limb CMEs have their core material concentrated along the line of sight, whereas west limb ones have it extended and perpendicular to the line of sight. Figure 15c presents the EUV SRs associated with the corresponding CMEs of Fig. 15b. The two 

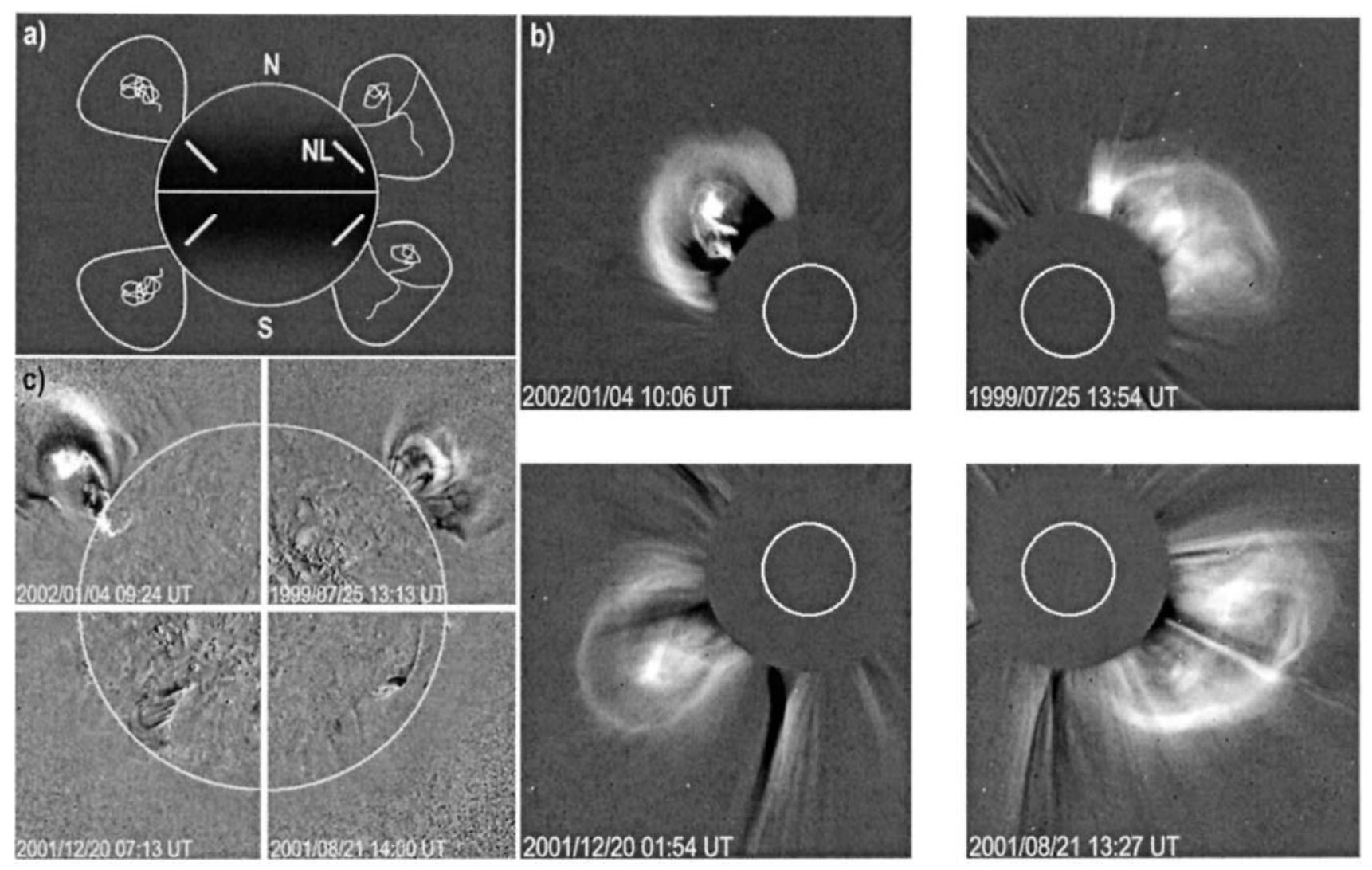

Fig. 15. a) Simplified scheme depicting the extreme cases of CME projections for front-side events. NL stands for neutral line. b) Examples of four projected LASCO C2 CMEs matching the basic scheme. c) Source region features of the CMEs shown in b). In the northern hemisphere, eruptive features are displayed, while the southern hemisphere shows post eruptive arcades.

cases in the northern hemisphere show eruptive features being self-similar to the CME structures observed later in C2, while the two southern ones depict post-eruptive arcades perpendicular to the limb in the East case and parallel to it in the West case. It must be noted that the described picture in Fig. 15 reverses for far-sided CMEs near the limb (Fig. 16) because the orientation of the neutral lines with respect to the observer is reversed in this case.

Figure 15 presents an ideal view based on cases with typical hemispheric orientations of the SRs. However, in reality the variety of inclinations of the SRs, from almost horizontal to nearly vertical ones as shown in Fig. 10a, yield many cases in which the structure of the CMEs in $\mathrm{C} 2$ will be a combination of the two basic views. The intermediate cases as well will range from being purely aligned along the line of sight or perpendicular to it. However, under general circumstances, the topology of the resulting CME can be considered as consistent with the orientation of the neutral line and its position on the solar disk. Column 13 of Table 1 specifies for each event whether the orientation of the SR matched the expected CME shape approximated by a straight or curved axis of an imaginary cylinder. For 94 out of 124 cases (76\% of the selected structured CMEs) such an agreement was found. This finding suggests that CMEs can be interpreted as three-dimensional magnetic field entities whose global configuration agrees with the characteristics of the underlying photospheric SR geometry with its loops or flux ropes expanded into the low corona. It should be noted here that this interpretation is independent of the existence of U-shaped white-light features, considered by some authors as to outline the flux rope when it is oriented along the line of sight (Chen et al. 1997; Wood et al. 1999). Because of the concave up nature of magnetic field lines in flux ropes, in principle U-shaped features may also be visible for flux ropes highly inclined with respect to the line of sight (Gibson \& Low 2000), as in the event of Fig. 2.

The neutral line location and its orientation play a crucial role in determining the topology of the projected CME in $\mathrm{C} 2$. However, other factors also affect the topology of CMEs as experienced from the inspection of the collection of structured events, a finding that could be anticipated from the unified scheme depicted for structured CMEs in Fig. 15 if one takes variations of the initial conditions into account. The neutral lines often can not be represented by fairly straight lines, the loops above them may have undergone considerable shear motions, the tilts of the neutral lines with respect to the NS line scatter, amongst other possible complicating factors. Altogether these factors, which are apparent from solar observations, imply the existence of complicated configurations and deviations from the basic CME scheme. Further, prominences do not always rise uniformly. Sometimes one of the legs seems to detach and rise before the rest of the prominence material lifts off (see e.g., CME on 2nd November 2000), leading 


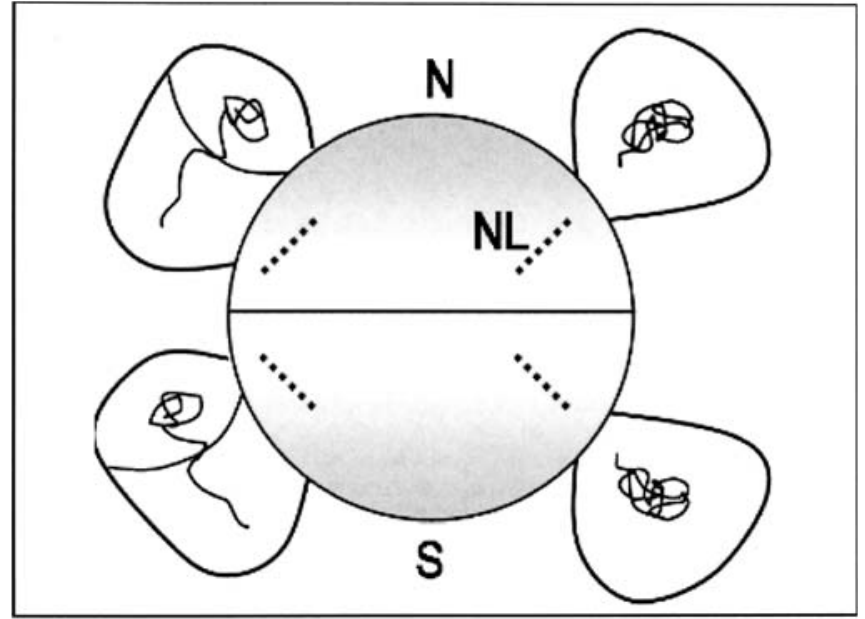

Fig. 16. Same as Fig. 15a, but for backside events. The neutral lines are dotted in this case, symbolising their far-side locations.

eventually to alterations of the expected $\mathrm{C} 2$ white-light profile. On the whole, the dynamics of prominences can exhibit various levels of complexity (e.g. Tandberg-Hanssen 1974).

In general the events presented in Table 1 reveal that the degree of symmetry depends on the length of the part of the neutral line from which the associated structured CMEs originated. Therefore the cylindrical geometry presented in Fig. 15 especially matched CMEs with large SR lengths which are typically found in decaying regions that have spread to higher latitudes (see Fig. 9 for comparison). On the contrary, CMEs associated with shorter neutral lines, especially those of newly developed compact active regions, showed a lower degree of correspondence. Ideally, the CME scheme implies a relationship between the length of the SR and the $A W$ of the corresponding CME. To investigate this dependence, the SR lengths for neutral lines closely parallel to the west limb events indicated by a superscript $b$ in Table 1), expected to yield CMEs with their core material aligned perpendicularly to the line of sight, were compared with the respective $A W \mathrm{~s}$ (Fig. 17). The data points show considerable scatter, and a linear fit yields the following correspondence: $A W=0.84$ Length $_{\mathrm{SR}}+79$. The scatter of the data points seems obvious if one takes into account that projection effects in the measured $A W \mathrm{~s}$ of the CMEs are still present. Another aspect can lead to a dilution of the trend, because the longest SRs - typically horizontal, and therefore not parallel to the limb - are found in magnetically decaying old regions which were apparently not producing wide spreading CMEs whereas some small source regions did produce some very impulsive wide spreading CMEs.

As a CME propagates outward it will interact with the ambient corona which can lead to significant changes of its shape as experienced from the distorted profile of some of the events listed in Table 1. Due to these possible interactions, not only the shape can undergo modifications, but CMEs often appeared at different PAs with respect to the SR locations. To study this effect, the central PAs of the CMEs were compared with those of the SRs (Col. 11 in Table 1), measured by taking the midpoint of each SR. As shown in Fig. 18, the CME occurrence frequencies (rectangular columns) peak near the equatorial regions at

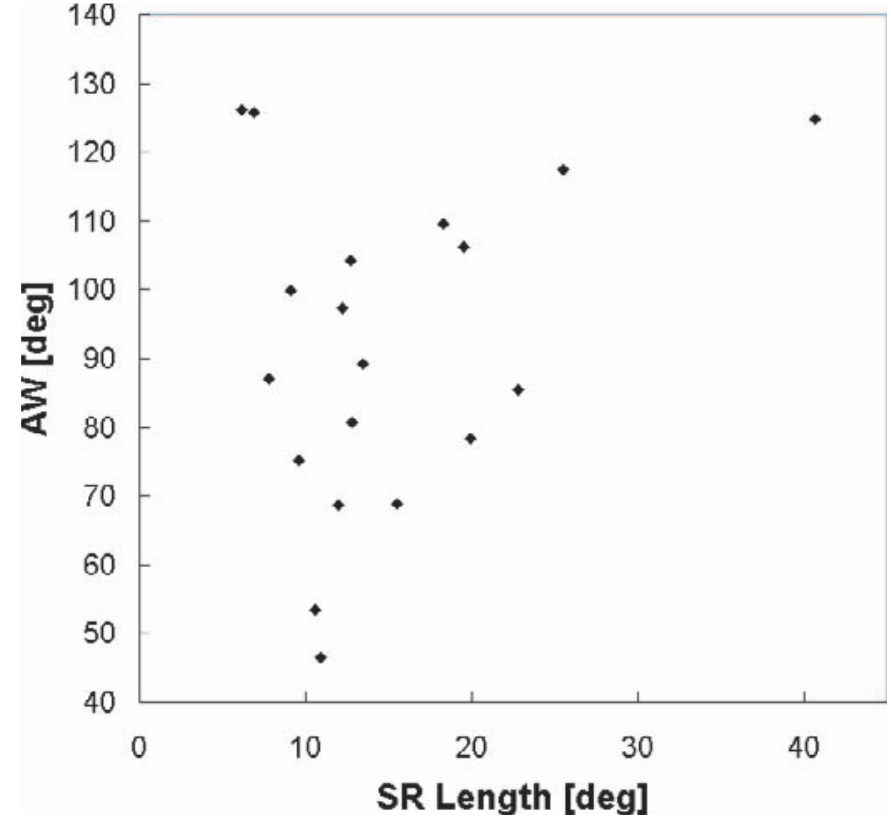

Fig. 17. Angular Width of structured CMEs as a function of the associated SR Length for cases in which the neutral line was parallel to the line of the limb.

$\mathrm{PA}=90^{\circ}$ and $\mathrm{PA}=270^{\circ}$ for the east and west limb respectively, despite the fact that the SRs (solid line) are concentrated in the two activity belts. If one splits the columns into times of lower (1996-1998) and higher (1999-2002) solar activity periods, a two peak behaviour arises in the CME PA distribution, similar to that of the SRs. The confinement of CMEs to equatorial regions in times of solar minimum, as well as the spread over all PAs during solar maximum, has also been observed in the Solwind (Howard et al. 1985, 1986), the SMM (Hundhausen 1993) and the MK3 (St. Cyr et al. 1999) datasets. During solar minimum any solar wind outflow from the activity belts in each hemisphere is usually embedded in fast solar wind emanating from large polar coronal holes (e.g. Bothmer 1999). CMEs that originate during these times from the solar activity belts, and which possess a total plasma and magnetic field pressure not exceeding that of the surrounding flow, will get deflected towards lower latitudes.

The CME deviation $\delta$ (Col. 12 in Table 1) with respect to the SR PA is shown in Fig. 19 for each event of Table 1 together with the total area of coronal holes during the corresponding Carrington Rotation as provided by the Kitt Peak He $10830 \AA$ data. Positive values of the deflection angle $\delta$ correspond to equatorward deviated CMEs, while negative ones correspond to poleward deviated CMEs. At least until mid 1998 the CMEs were systematically deflected towards the equator in both hemispheres, unfortunately the second half of 1998 was disrupted by the SOHO recovery phase. The correspondence of the systematic deflection of CMEs towards the equator with the total coronal hole area in time is striking until solar maximum and supports the assumption that coronal holes in the vicinity of CME SRs can play a major role in this respect. The average deflection $\delta$ for this time period was $18.6^{\circ}$. From January 2000 until the end of 2002, the direction of deflection shows no 


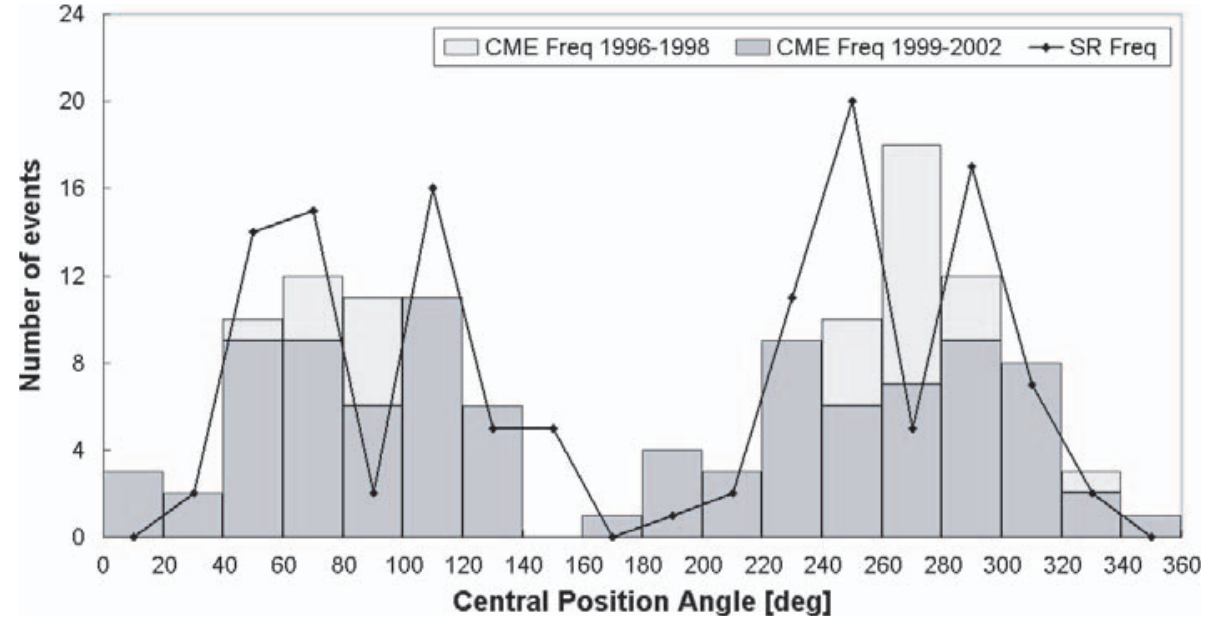

Fig. 18. Distribution histogram of the CME central position angles for the 124 structured events during 1996-2002, with identified source regions (columns), in comparison with the SRs central PA (solid line) displayed in bins of $20^{\circ}$. The CME PAs have been split in two time periods: 1996-1998 (light gray columns) and 1999-2002 (dark gray columns). Note the change in the SR PA distribution from double to single peak.

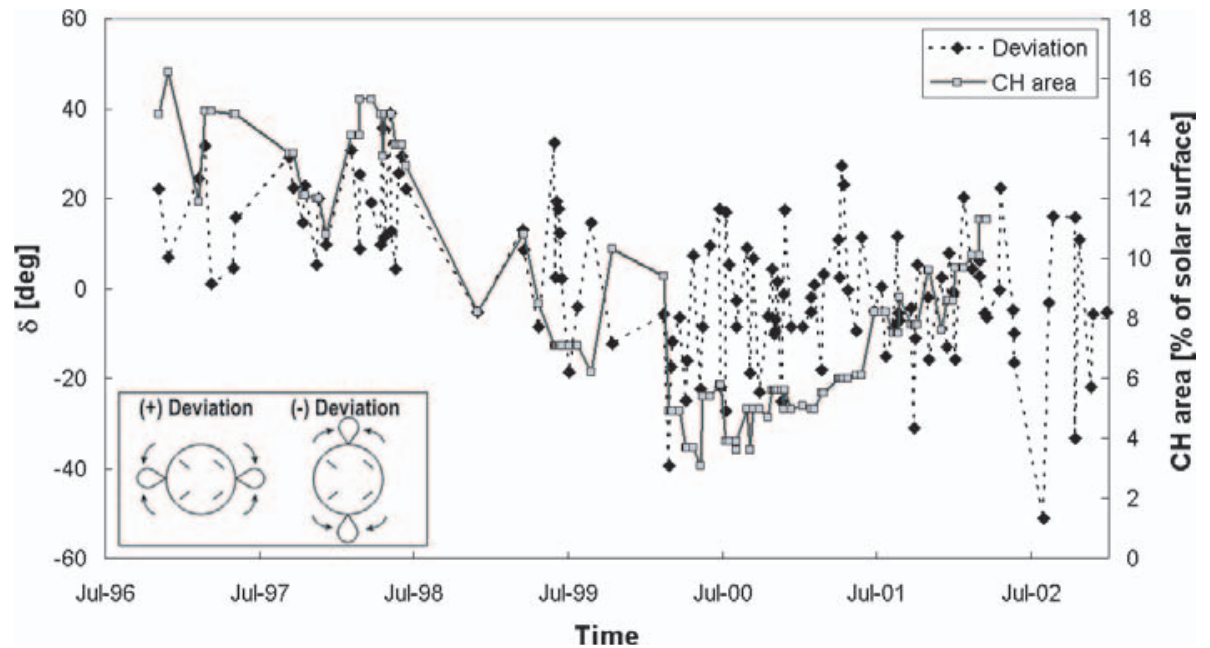

Fig. 19. Deviation angle $\delta$ for each structured CME with respect to its associated source region as a function of time (dashed line with diamonds). CME deviation towards the solar equator corresponds to positive values of $\delta$, while deviations towards the poles correspond to negative ones. The time evolution of the total area of coronal holes on the Sun, as provided by Kitt Peak He $10830 \AA$ data, is shown for comparison for the corresponding Carrington Rotations (solid line with squares). systematic trend, with $\langle\delta\rangle=-4.4^{\circ}$. CMEs deflected towards the equator showed commonly a smaller deviation angle and a number of CMEs was almost not deflected at all. Especially at times of solar activity maximum in 2000 , about two thirds of the CMEs were deflected rather towards the poles, with $\delta$ oscillating around $-7.1^{\circ}$. It appears as if the year 1999 seemed to be an intermittent time period concerning the deflection of CMEs.

\section{Summary and conclusions}

From a survey of SOHO/LASCO C2 observations taken from 1996 until the end of 2002 we have compiled a set of 276 structured CMEs (available upon request to the authors), as defined in Sect. 3, to infer observational properties that help to resolve their three-dimensional configuration. This group of CMEs exhibited clear features, and were for this reason expected to originate from limb or near limb regions. Out of this set, for 124 structured events presented in Table 1 the low coronal and underlying photospheric SRs could be identified through the analysis of SOHO EIT and MDI as well as ground-based $\mathrm{H} \alpha$ data. Basic properties of these CMEs, such as $A W \mathrm{~s}$ and PAs were recalculated in order to properly compare them with the heliographic positions, lengths and inclinations of the SRs. The results provided by the detailed analysis of the 124 structured CMEs can be summarized as follows:

a) The frequency distribution in time of structured CMEs followed the sunspot cycle during the investigated time period (1996-2002), in close correspondence with the total number of CMEs except in 2002.

b) The majority (97 events, 78\%) of structured CMEs originated from limb (65 events, 52\%) and near limb (32 events, 26\%) source regions, the rest had source regions located farther away from the limb than $40^{\circ}$.

c) All structured CMEs arose from regions of opposite magnetic polarities, of considerably smaller spatial scales than the corresponding CMEs, indicative for the expansion of low coronal magnetic structures into the FOV of $\mathrm{C} 2$.

d) The source regions were located primarily in the activity belts in both hemispheres, with typical lengths of $5^{\circ}-20^{\circ}$, while the longer ones were found at higher latitudes.

e) The inclination angle $\gamma$ of the neutral lines with respect to the NS-line varied both with heliographic latitude and with time, with a tendency for lower inclinations being most frequent in the second half of 2000 , i.e. at times of the polarity reversal of the Sun's global magnetic field. The inclination $\gamma$ commonly increased with latitude, i.e. became more parallel to the equator. 
f) The 3D topology of structured CMEs observed in the field of view of LASCO C2 (commonly matching the typical three-part CME structure) can be classified according to a basic scheme (see Fig. 15) in which the fundamental parameters are the heliographic position and orientation of the source region's neutral line, separating opposite magnetic polarities.

g) The apparent profile of a CME may differ more or less from the basic scheme because of the solar variability of the fundamental parameters, e.g. many neutral lines are not straight lines, but have rather complicated topologies, especially in active regions. The degree of correspondence with the scheme also depends on the absolute values of the SR lengths which will impose difficulties for small values typically found in compact active regions.

h) The central PA of the structured CMEs was systematically deviated with respect to that of the SRs by about $20^{\circ}$ to lower latitudes during the years after solar minimum (1996-1998). The deflection of structured CMEs is most likely due to the fast solar wind flow from polar coronal holes that encompasses the CMEs' expansion at the higher latitudes. At times of higher solar activity the deviations vary in correspondence with the complexity of the corona.

Even though CMEs were first interpreted as three-dimensional structures (Crifo et al. 1983; Fisher \& Munro 1984) rather than two-dimensional loops (Trottet \& MacQueen 1980) many years ago, they are still thought to be balloon or bubble-like structures with a high degree of spherical symmetry. However, the results of this detailed study of CMEs imply that their $3 \mathrm{D}$ structure is organized along an axial direction, which is often directly visible in the case of extended prominences, shaped by the neutral line separating regions of opposite magnetic field polarity in the CME's source region.

The real axis of the CME seems to correspond with the long axis of a large-scale helical magnetic flux rope that was formed in the SR, with the prominence being the bottom part of this magnetic system. The shape of the neutral line is often, but not always fully aligned with the prominence axis. During the eruption of the flux rope it may or may not undergo considerable distortion depending on the level of complexity of its evolution.

The CME as represented by a magnetic flux rope formed in the low corona before its eruption will suffer an enormous expansion once it is triggered to lift off (see theories on CME initiation in e.g. Forbes 2000) as supported by the often directly visible self-similarity of the magnetic features in the studied events, as seen in the low corona and further out. The expansion of CMEs is most dramatic within the first two solar radii and likely the reason why "global CMEs" (e.g. Brueckner 1996; Brueckner et al. 1998) seem just to be caused by projection effects. Ultimately, the expanding flux rope CME may be identified as a magnetic cloud in the solar wind (e.g. Bothmer $\&$ Schwenn 1998). The 3D topology of CMEs derived from this study and the validity of the general scheme presented can be directly proven by means of multipoint observations. The NASA STEREO mission, to be launched in 2005, will provide for the first time two observations of the same CME from two different points, enabling the direct proof of these results. In the meantime the results can be used for the development of 3D synthetic CMEs which illustrate the two views as perceived from STEREO.

Acknowledgements. We are thankful to Prof. Dr. Sami Solanki and Prof. Dr. Rainer Schwenn for valuable discussions. The useful comments and suggestions provided by Dr. Sarah Gibson have greatly improved the quality of the manuscript. This work is part of the scientific investigations of the project Stereo/Corona, in context of the International Max Planck Research School, supported by the German "Bundesministerium für Bildung und Forschung" through the "Deutsche Zentrum für Luft- und Raumfahrt e.V." (DLR, German Space Agency) under project number 50 OC 0005. Stereo/Corona is a science and hardware contribution to the optical imaging package SECCHI, currently being developed for the NASA STEREO mission to be launched in 2005. Further information can be found at http://stp.gsfc.nasa.gov/missions/stereo/stereo.htm. We thank all the members of the SOHO/LASCO/EIT/MDI consortium who built the instruments and provided the data used in this study. LASCO, EIT and MDI images are courtesy of SOHO consortium. SOHO is a project of international cooperation between ESA and NASA. The CME catalog is generated and maintained by NASA and The Catholic University of America in cooperation with the Naval Research Laboratory. We aknowledge the use of $\mathrm{H} \alpha$ data from the Observatory of Meudon and Big Bear Solar Observatory, as well as data from Yohkoh/SXT.

\section{References}

Amari, T., Luciani, J. F., Mikic, Z., \& Linker, J. 2000, ApJ, 529, L49 Benevolenskaya, E. E., Kosovichev, A. G., Lemen, J. R., Scherrer, P. H., \& Slater, G. L. 2002, ApJ, 571, L181

Bothmer, V., \& Schwenn, R. 1998, Ann. Geophys., 16, 1

Bothmer, V., Bougeret, J.-L., Cargill, P., et al. 1998, in Proc. of Crossroads for European Solar \& Heliospheric Physics (Noordwijk: ESA), 145

Bothmer, V. 1999, in Proc. of ESA Workshop on Space Weather 1998 (Noordwijk: ESA), 117

Brueckner, G. E., Howard, R. A., Koomen, M. J., et al. 1995, Sol. Phys., 162, 357

Brueckner, G. E. 1996, EOS, Trans. Am. Geophys. Union, 77, S204.1

Brueckner, G. E., Delaboudinière, J.-P., Howard, R. A., et al. 1998, Geophys. Res. Lett., 25, 3019

Chen, J., Howard, R. A., Brueckner, G. E., et al. 1997, ApJ, 490, L191

Crifo, F., Picat, J. P., \& Cailloux, M. 1983, Sol. Phys., 83, 143

Delaboudinière, J.-P., Artzner, G. E., Brunaud, J., et al. 1995, Sol. Phys., 162, 291

Dere, K. P., Brueckner, G. E., Howard, R. A., \& Michels, D. J. 1999, ApJ, 516, 465

Fisher, R. R., \& Munro, R. H. 1984, ApJ, 280, 428

Fleck, B., Domingo V., \& Poland, A. I. 1995, The SOHO Mission (Dordrecht: KAP)

Forbes, T. G. 2000, J. Geophys. Res., 105, 23153

Gibson, S. E., \& Low, B. C. 1998, ApJ, 493, 460

Gibson, S. E., \& Low, B. C. 2000, J. Geophys. Res., 105, 18187

Gopalswamy, N., Lara, A., Yashiro, S., Nunes, S., \& Howard, R. A. 2003, in Proc. ISCS 2003 Symp., Solar Variability as an Input to the Earth's Environment, ed. A. Wilson (Noordwijk: ESA), 403

Gosling, J. T., Hildner, E., MacQueen, R. M., et al. 1974, J. Geophys. Res., 79, 4581 
Hale, G. E., Ellerman, F., Nicholson, S. B., \& Joy, A. H. 1919, ApJ, 49, 153

Howard, R. A., Sheeley, N. R., Jr., Koomen, M. J., \& Michels, D. J. 1985, J. Geophys. Res., 90, 8173

Howard, R. A., Sheeley, N. R., Jr., Koomen, M. J., \& Michels, D. J. 1986, The Solar Cycle Dependence of Coronal Mass Ejections, in The Sun and the Heliosphere in Three Dimensions, ed. R. G. Marsden (Norwell, Mass.: D. Reidel), 107

Howard, R. A., Brueckner, G. E., St. Cyr, O. C., et al. 1997, Observations of CMEs from SOHO/LASCO, in Coronal Mass Ejections, ed. N. Crooker, J. A. Joselyn, \& J. Feynman (Washington, DC: AGU), 17

Hudson, H. S., Lemen, J. R., St. Cyr, O. C., Webb, D. F., \& Sterling, A. C. 1998, GRL, 25, 2481

Hundhausen, A. J., Sawyer, C. B., House, L., Illing, R. M. E., \& Wagner, W. J. 1984, J. Geophys. Res., 89, 2639

Hundhausen, A. J. 1993, J. Geophys. Res., 98, 13177

Hundhausen, A. J. 1999, Coronal Mass Ejections, in The Many Faces of the Sun, ed. K. T. Strong, et al. (New York: Springer), 143

Illing, R. M. E., \& Hundhausen, A. J. 1985, J. Geophys. Res., 90, 275

Low, B. C. 2001, J. Geophys. Res., 106, 25141

MacQueen, R. M. 1993, Sol. Phys., 145, 169

Michalek, G., Gopalswamy, N., \& Yashiro, S. 2003, ApJ, 584, 472

Munro, R. H., Gosling, J. T., Hildner, E., et al. 1979, Sol. Phys., 61, 201

Plunkett, S. P., Vourlidas, A., Simberová, S., et al. 2000, Sol. Phys., 194, 371

Rust, D. M. 1998, in Proc. of Crossroads for European Solar \& Heliospheric Physics (Noordwijk: ESA), 133
Scherrer, P. H., Bogart, R. S., Bush, R. I., et al. 1995, Sol. Phys., 162, 129

Schwenn, R. 1986, SSR, 44, 139

St. Cyr, O. C., Burkepile, J. T., Hundhausen, A. J., \& Lecinski, A. R. 1999, J. Geophys. Res., 104, 12493

St. Cyr, O. C., Howard, R. A., Sheeley, N. R., et al. 2000, J. Geophys. Res., 105, 169

Srivastava, N., Schwenn, R., Inhester, B., Martin, S. F., \& Hanaoka, Y. 2000, ApJ, 534, 468

Sterling, A. C., Hudson, H. S., Thompson, B. J., \& Zarro, D. M. 2000, ApJ, 532, 628

Subramanian, P., \& Dere, K. P. 2001, ApJ, 561, 372

Tandberg-Hanssen, E. 1974, Solar Prominences (Dordrecht: Reidel Publishing Company)

Thompson, B. J., Plunkett, S. P., Gurman, J. B., et al. 1998, Geophys. Res. Lett., 25, 2465

Tousey, R., Howard, R. A., \& Koomen, M. J. 1974, BAAS, 6, 295

Tripathi, D., Bothmer, V., \& Cremades, H. 2004, A\&A, 422, 337

Trottet, G., \& MacQueen, R. M. 1980, Sol. Phys., 68, 177

Vourlidas, A., Subramanian, P., Dere, K. P., \& Howard, R. A. 2000, ApJ, 534, 456

Webb, D. F., \& Hundhausen, A. J. 1987, Sol. Phys., 108, 383

Webb, D. F. 1988, ApJ, 93, 1749

Webb, D. F. 2000, IEEE Trans. Plasma Science, 28, 1795

Wood, B. E., Karovska, M., Chen, J., et al. 1999, ApJ, 512, 484

Yashiro, S., Gopalswamy N., Michalek, G., et al. 2003, ApJ, submitted

Zhao, X. P., Plunkett, S. P., \& Liu, W. 2002, J. Geophys. Res., 107, 10.1029/2001JA009143 